\title{
BRANCHED CIRCLE PACKINGS AND DISCRETE BLASCHKE PRODUCTS
}

\author{
TOMASZ DUBEJKO
}

\begin{abstract}
In this paper we introduce the notion of discrete Blaschke products via circle packing. We first establish necessary and sufficient conditions for the existence of finite branched circle packings. Next, discrete Blaschke products are defined as circle packing maps from univalent circle packings that properly fill $D=\{z:|z|<1\}$ to the corresponding branched circle packings that properly cover $D$. It is verified that such maps have all geometric properties of their classical counterparts. Finally, we show that any classical finite Blaschke product can be approximated uniformly on compacta of $D$ by discrete ones.
\end{abstract}

\section{INTRODUCTION}

Circle packings are collections of circles in the plane with prescribed patterns of tangencies encoded in simplicial 2-complexes. They were proposed for discrete approximation of conformal mappings by Thurston in [T1]. A proof of his conjecture, in the case of hexagonal packings, was given by Rodin and Sullivan in [RS]. Shortly after that, Beardon and Stephenson suggested that circle packings can serve as discrete analogues of analytic functions and can be used to construct discrete parallels of classical results from complex analysis [BSt1]. In particular, they raised a question about existence of branched circle packings and discrete versions of analytic functions with branch points. In this paper we address their question. Although our answer does not settle the problem entirely, it gives complete solution for special classes of packings and functions. These classes are finite circle packings and finite Blaschke products, respectively. We first establish necessary and sufficient conditions for the existence of finite branched circle packings subject to given patterns of tangencies and given branch sets (Theorem 2.2). We also show (Theorem 2.5) that the boundary value problem for branched circle packings is always uniquely solvable, in both euclidean and hyperbolic geometries, as long as the conditions for the existence are satisfied. Although there are related results obtained by Bowers [B] and Garrett [G], our work is independent and yields more natural and easily checked conditions for the existence. In addition, techniques used in [B] and $[G]$ do not extend to the infinite case, while our methods extend giving a

Received by the editors October 21, 1993 and, in revised form, November 18, 1994.

1991 Mathematics Subject Classification. Primary 30D50, 30G25, 30C60.

Key words and phrases. Circle packing, Blaschke products, discrete analytic functions.

The author gratefully acknowledges support of the National Science Foundation and the Tennessee Science Alliance. 
proof of the existence of infinite branched circle packings in the forthcoming paper [D].

Once finite branched circle packings are in place, we introduce the notion of discrete Blaschke products as circle packing maps with the domain packings being Andreev packings and with the range packings being Bl-type packings (branched versions of Andreev packings). We verify (Theorem 3.3) that such maps are compositions of self-homeomorphisms of the unit disc followed by classical Blaschke products.

The last section of our paper is devoted to the approximation results for discrete Blaschke products. In that section we generalize Rodin and Sullivan Length-Area Lemma [RS] to the branched case (see the proof of Lemma 4.5.2) and we show "K-quasiregularity" of certain families of branched circle packings (Lemma 4.6.1). Finally, using arguments from the theory of qusiregular mappings, we prove our main result (Theorem 4.7.3) which states that any classical finite Blaschke product can be approximated uniformly on compacta of the unit disc by discrete ones. We want to point out that our proof of Theorem 4.7.3 contains a practical method for the construction of an approximating sequence of discrete Blaschke products, which has already found its application in [DSt].

This paper is a part of the author's Ph.D. thesis. The author would like to express his appreciation to Professor Ken Stephenson, the thesis advisor, for the guidance and attention he provided. The author is also very grateful to Professors Robert Daverman, Klaus Johannson, and Carl Sundberg for stimulating discussions.

\section{Preliminaries}

We are going to introduce briefly some terminology and definitions related to circle packings. For a more complete picture we refer the reader to [BSt1] or [DSt].

1. Packings. Let $\mathbf{T}$ be a (locally finite) triangulation of a disc such that $\mathbf{T}$ is finite when the disc is closed and infinite without boundary when the disc is open. We will be assuming that $\mathbf{T}$ has an orientation induced from an orientation of the disc. The sets of vertices, boundary vertices, and interior vertices of $\mathbf{T}$ will be denoted by $V(\mathbf{T})$, bd $\mathbf{T}$, and int $\mathbf{T}$, respectively.

Let $P$ be a collection of circles in the plane. Then we say that $P$ is a circle packing for $\mathbf{T}$ if:

- there exists a 1-to-1 correspondence between the vertices $v \in V(\mathbf{T})$ and the circles $C_{P}(v)$ of $P$ such that circles $C_{P}(u)$ and $C_{P}(w)$ are externally tangent whenever $u$ and $w$ are vertices sharing an edge in the complex $\mathbf{T}$,

- $P$ is orientation preserving, i.e. if $v_{1}, v_{2}, v_{3}$ are the vertices of a face in $\mathbf{T}$ taken in the positive order (with respect to the orientation of T) then $C_{P}\left(v_{1}\right), C_{P}\left(v_{2}\right), C_{P}\left(v_{3}\right)$ form a positively oriented triple of circles in the plane.

If $P$ has all its elements contained in the unit disc $\Delta=\{|z|<1\}$ then it can be viewed as a circle packing in the hyperbolic plane (i.e., in $\Delta$ with the hyperbolic metric), and in such case we consider $P$ as a hyperbolic circle packing.

A flower in $P$ associated with a vertex $v \in V(\mathbf{T})$ is a collection of the 
following circles from $P$ : the circle $C_{P}(v)$, called the center, and circles that correspond to the neighboring vertices of $v$, called the petals. The number of petals is the degree of $v$.

If a circle packing has the property that all its circles have mutually disjoint interiors then it is called univalent. The reader should be aware of the fact that for any triangulation $\mathbf{T}$ of a disc there exists a univalent circle packing associated with $\mathbf{T}$ ([BSt1]). If $\mathbf{T}$ is finite then there is an important class of univalent circle packings determined by $\mathbf{T}$ called Andreev packings. These are packings contained in the unit disc such that their boundary circles are internally tangent to the unit circle. Among univalent circle packings associated with infinite triangulations there is one which will be of special interest for us. Its complex is an infinite constant 6-degree complex identified with an infinite regular hexagonal lattice in $\mathbf{C}$, and the packing itself is a regular hexagonal circle packing in $\mathbf{C}$ with all circles having the same radii.

2. Branched packings. If $P$ is a circle packing for $\mathbf{T}$ then a function $r$ : $V(\mathbf{T}) \rightarrow(0, \infty)$ which assigns to each vertex in $V(\mathbf{T})$ the euclidean radius of the corresponding circle in $P$ is called the radius function of $P$. The function $r$ determines for each interior vertex of $\mathbf{T}$ its angle sum in the following way: Write $s_{P}(u)$ for the center of $C_{P}(u)$ and let $v \in$ int $\mathbf{T}$, then the angle sum $\theta_{r}(v)$ at $v$ is $\sum \alpha_{r}(v, \triangle)$, where the summation is over all faces $\triangle$ in the star of $v$ in $\mathbf{T}$, and $\alpha_{r}(v, \triangle)=\alpha_{r}\left(v, \Delta\left(v, u_{1}, u_{2}\right)\right)$ is the angle at a vertex $s_{P}(v)$ in the euclidean triangle $\triangle s_{P}(v) s_{P}\left(u_{1}\right) s_{P}\left(u_{2}\right)$ with the vertices $s_{P}(v), s_{P}\left(u_{1}\right)$, $s_{P}\left(u_{2}\right)$.

It follows almost immediately from the definition of a circle packing that all angle sums of $P$ are positive integer multiples of $2 \pi$. A vertex in $\mathbf{T}$ with the angle sum determined by $P$ equal to $2 n \pi, n \geq 2$, is called a branch point (vertex) of $P$ of multiplicity $n$. If we want to stress that a circle packing has (does not have) a branch point then we refer to it as a branched (locally univalent) circle packing. Notice that univalent circle packings are necessarily locally univalent.

3. Mappings. A circle packing $P$ for $\mathbf{T}$ naturally induces a simplicial function $\mathscr{S}_{P}: \mathbf{T} \rightarrow \mathbf{C}$ which maps each vertex of $\mathbf{T}$ to the (euclidean) center of the corresponding circle in $P$ and then is extended via barycentric coordinates to edges and faces of $\mathbf{T}$. The geometric complex $\mathscr{S}_{P}(\mathbf{T})$ is called the (euclidean) carrier of $P$ and denoted $\operatorname{carr}(P)$. If $P$ is univalent then the induced simplicial map $\mathscr{S}_{P}$ is an embedding and the carrier of $P$ is a subset of $\mathbf{C}$ equal to the union of images of all faces of $\mathbf{T}$ under $\mathscr{S}_{P}$.

Let $Q$ be another circle packing for $\mathbf{T}$, and let $r_{P}$ and $r_{Q}$ denote the radius functions for packings $P$ and $Q$, respectively. Then the map $G_{Q, P}:=\mathscr{S}_{P}$ 。 $\mathscr{S}_{Q}^{-1}: \operatorname{carr}(Q) \rightarrow \operatorname{carr}(P)$ is called the simplicial map from $Q$ to $P$. The map $G_{Q, P}^{\#}: \operatorname{carr}(Q) \rightarrow(0, \infty)$ defined by $G_{Q, P}^{\#}\left(\mathscr{S}_{Q}(v)\right)=r_{P}(v) / r_{Q}(v)$ on the set of vertices of $\mathscr{S}_{Q}(\mathrm{~T})$ and then extended affinely to faces of $\mathscr{S}_{Q}(\mathrm{~T})$ is called the ratio function from $Q$ to $P$ (cf. [RS], [DSt]).

4. Radii and angle sums. One way of working with circle packings is by working with their radius functions. Such an approach allows for certain generalizations. In particular, for any function $\rho: V(\mathbf{T}) \rightarrow(0, \infty)$ (an "abstract" radius function) we can define an "abstract" angle sum at any interior vertex of T. To achieve this we do the following construction: 
Let $\triangle=\triangle\left(u_{0}, u_{1}, u_{2}\right)$ be the face in $\mathbf{T}$ with the vertices $u_{0}$, $u_{1}, u_{2}$ listed in the positive order. Let $C\left(u_{0}\right), C\left(u_{1}\right), C\left(u_{2}\right)$ be a positively oriented triple of mutually and externally tangent circles in the plane with radii $\rho\left(u_{0}\right), \rho\left(u_{1}\right), \rho\left(u_{2}\right)$ and centers $s\left(u_{0}\right), s\left(u_{1}\right)$, $s\left(u_{2}\right)$, respectively. Write $\alpha_{\rho}\left(u_{j}, \triangle\left(u_{0}, u_{1}, u_{2}\right)\right), j=0,1,2$, to denote the angle at the vertex $s\left(u_{j}\right)$ in the triangle $\triangle s\left(u_{0}\right) s\left(u_{1}\right) s\left(u_{2}\right)$. For $v \in$ int $\mathbf{T}$ we define $\theta_{\rho}(v):=\sum \alpha_{\rho}(v, \triangle)$, where the summation is over all faces $\Delta$ in the star of $v$ in $\mathbf{T}$. Then $\theta_{\rho}(v)$ is called the ("abstract") angle sum at $v$ determined by $\rho$.

It is almost immediate from the above construction that if $\rho$ happens to be the radius function $r_{P}$ of a circle packing $P$ for $\mathbf{T}$ then $\theta_{\rho}(v)=\theta_{r_{P}}(v)$ for any $v \in$ int $\mathbf{T}$. It is common, therefore, to refer to a collection $\{\rho(v)\}_{v \in V(\mathbf{T})}$ as a collection of radii, even if $\rho: V(\mathbf{T}) \rightarrow(0, \infty)$ is not the radius function for any circle packing for $\mathbf{T}$. Necessary and sufficient conditions for this are easily established in terms of angle sums: $\rho: V(\mathbf{T}) \rightarrow(0, \infty)$ is the radius function for some circle packing for $\mathbf{T}$ if and only if all values of $\theta_{\rho}:$ int $\mathbf{T} \rightarrow(0, \infty)$ are multiples of $2 \pi$. This depends on the fact that $\mathbf{T}$ is "simply connected" and was proven in [BSt2] for $\theta_{\rho} \equiv 2 \pi$; the proof can easily be extended for values of $\theta_{\rho}$ which are integral multiples of $2 \pi$. In such case, that is when the values of $\theta_{\rho}$ are multiples of $2 \pi$, a circle packing whose radius function is $\rho$ is called a circle packing induced by $\rho$. Such a circle packing is determined uniquely by the location in the plane of a pair of its tangent circles associated with a given edge of $\mathbf{T}$, i.e., if $u_{1} u_{2}$ is an edge in $\mathbf{T}$, and $P=\{C(u)\}_{u \in V(\mathbf{T})}$ and $P^{\prime}=\left\{C^{\prime}(u)\right\}_{u \in V(\mathbf{T})}$ are circle packings induced by $\rho$ such that $C\left(u_{j}\right)=C^{\prime}\left(u_{j}\right)$ for $j=1,2$, then $P=P^{\prime}$. In other words, any circle packing induced by $\rho$ is unique up to rigid motions.

Whether or not $\rho$ is a radius function, for $w \in$ bd $\mathbf{T}$ we define the interior angle $\gamma_{\rho}(w)$ at $w$ by $\gamma_{\rho}(w):=\sum \alpha_{\rho}(v, \triangle)$, where the summation is over all faces $\triangle$ in $\mathbf{T}$ with the vertex $w$. Then $\theta_{\rho}(\cdot)$ and $\gamma_{\rho}(\cdot)$ are related by the following euclidean version of the Gauss-Bonnet formula

$$
\sum_{v \in \text { int } \mathbf{T}}\left(2 \pi-\theta_{\rho}(v)\right)=2 \pi-\sum_{w \in \text { bd } \mathbf{T}}\left(\pi-\gamma_{\rho}(w)\right)
$$

Finally, we would like to mention some monotonicity properties of $\theta_{\rho}(\cdot)$. Suppose $\rho^{\prime}, \rho: V(\mathbf{T}) \rightarrow(0, \infty)$ are such that $\rho^{\prime}\left(v_{0}\right)<\rho\left(v_{0}\right)$ for some $v_{0} \in$ int $\mathbf{T}$ and $\rho^{\prime} \equiv \rho$ in $V(\mathbf{T}) \backslash\left\{v_{0}\right\}$. Let $v_{0}, u_{1}, u_{2}$ be the vertices of a face in $\mathbf{T}$. Then $\alpha_{\rho^{\prime}}\left(v_{0}, \Delta\left(v_{0}, u_{1}, u_{2}\right)\right)>\alpha_{\rho}\left(v_{0}, \Delta\left(v_{0}, u_{1}, u_{2}\right)\right)$, and $\alpha_{\rho^{\prime}}\left(u_{j}, \triangle\left(v_{0}, u_{1}, u_{2}\right)\right)<\alpha_{\rho}\left(u_{j}, \triangle\left(v_{0}, u_{1}, u_{2}\right)\right)$ for $j=1,2$. In particular, $\theta_{\rho}\left(v_{0}\right)<\theta_{\rho^{\prime}}\left(v_{0}\right)$ and $\theta_{\rho}(v) \geq \theta_{\rho^{\prime}}(v)$ for $v \in$ int $\mathbf{T} \backslash\left\{v_{0}\right\}$. Moreover, if maps $\rho_{n}: V(\mathbf{T}) \rightarrow(0, \infty)$ are such that $\lim _{n \rightarrow \infty} \rho_{n}\left(v_{0}\right)=0\left(\lim _{n \rightarrow \infty} \rho_{n}\left(v_{0}\right)=\right.$ $\infty)$ and $\rho_{n} \equiv \rho$ in $V(\mathbf{T}) \backslash\left\{v_{0}\right\}$ then $\lim _{n \rightarrow \infty} \alpha_{\rho_{n}}\left(v_{0}, \triangle\left(v_{0}, u_{1}, u_{2}\right)\right)=\pi$ $\left(\lim _{n \rightarrow \infty} \alpha_{\rho_{n}}\left(v_{0}, \triangle\left(v_{0}, u_{1}, u_{2}\right)\right)=0\right)$.

Since our prime interest will be in euclidean circle packings, we have introduced radii and angle sums from the euclidean point of view, but the reader should be aware that similar constructions and properties as above can be achieved in the hyperbolic setting as well ([BSt2]). 


\section{FINITE BRANCHED CIRCLE PACKINGS}

Let $\mathbf{T}$ be a finite triangulation of a disc. Suppose that $\left\{v_{i}\right\}_{i=1}^{N}$ and $\left\{w_{i}\right\}_{i=1}^{M}$ are the sets of interior and boundary vertices of $\mathbf{T}$, respectively. Let $b_{1}, \ldots, b_{m}$ be designated interior vertices of $\mathbf{T}$. Suppose that $k_{1}, \ldots, k_{m}$ are positive integers.

In this section we want to construct a branched circle packing satisfying the following requirement:

$$
\begin{aligned}
\theta\left(b_{j}\right) & =\left(1+k_{j}\right) 2 \pi \quad \text { for } j=1, \ldots, m, \text { and } \\
\theta(v) & =2 \pi \text { for } v \in \operatorname{int} \mathbf{T} \backslash\left\{b_{1}, \ldots, b_{m}\right\},
\end{aligned}
$$

where $\theta(v)$ denotes the angle sum at the vertex $v$ determined by the collection of radii of circles from the packing. If there exists a circle packing $P$ that satisfies $(*)$ then $b_{j}$ will be called a branch vertex of multiplicity $1+k_{j}$ (order $\left.k_{j}\right)$ and $\left\{\left(b_{1}, k_{1}\right),\left(b_{2}, k_{2}\right), \ldots,\left(b_{m}, k_{m}\right)\right\}$ will be called the branch set for $\boldsymbol{P}$. If $k_{j}=1$ then $b_{j}$ will be called a simple branch vertex. If all branch vertices of $P$ are simple then we will write shortly $\operatorname{br}(P)=\left\{b_{1}, b_{2}, \ldots, b_{m}\right\}$.

Before we settle the existence question of branched circle packings we need the following definition, in which $\mathbf{N}$ denotes the set of positive integers.

Definition 1. A set $\mathfrak{U}=\left\{\left(u_{1}, l_{1}\right),\left(u_{2}, l_{2}\right), \ldots,\left(u_{n}, l_{n}\right)\right\} \subset($ int $\mathbf{T}) \times \mathbf{N}$ is called a branch structure for $\mathbf{T}$ if every simple closed edge-path $\Gamma$ in $\mathbf{T}$ has at least $2 \ell(\Gamma)+3$ edges, where $\ell(\Gamma)=\sum_{i=1}^{n} \delta_{i}(\Gamma) l_{i}$ and $\delta_{i}(\Gamma)$ is equal to 1 if $u_{i}$ is enclosed by $\Gamma$ and 0 otherwise.

We are now ready to state

Theorem 2. There exists an euclidean branched circle packing $P$ for $\mathbf{T}$ with the branch set $\mathfrak{B}=\left\{\left(b_{1}, k_{1}\right),\left(b_{2}, k_{2}\right), \ldots,\left(b_{m}, k_{m}\right)\right\}$ if and only if $\mathfrak{B}$ is a branch structure for $\mathbf{T}$.

Proof. We will first prove the existence part of the theorem. This will be done by introducing an algorithm for construction of a branched circle packing, and then by showing that such an algorithm really works.

Suppose $\mathfrak{B}$ is a branch structure for $\mathbf{T}$. Let $A$ be any locally univalent circle packing for $\mathbf{T}$ (e.g., an Andreev packing). Write $r(v)$ for the (euclidean) radius of the circle in $A$ associated with the interior vertex $v$, and write $R(w)$ for the (euclidean) radius of the circle in $A$ associated with the boundary vertex $w$. Let $\Phi(v)$ be the angle sum at $v$ in $A$, i.e., $\Phi(v)$ is the angle sum at $v(=2 \pi)$ determined by the collection of radii $\left\{r\left(v_{i}\right)\right\}_{i=1}^{N} \cup\left\{R\left(w_{i}\right)\right\}_{i=1}^{M}$.

We now proceed to describe an algorithm for construction of a branched circle packing determined by $\mathbf{T}$ and the branch set $\mathfrak{B}$. The algorithm starts with the radii of $A$, proceeds through a sequence of loops and their outputs, and ends with a collection of limit radii. In each loop we will successively adjust radii of all interior circles, and the results of adjustments will be called the output of the loop. The boundary radii $R\left(w_{i}\right)$ will remain unchanged throughout. The loops are going to be defined inductively as follows.

$\operatorname{Loop}(0)$ : We set $r_{0}\left(v_{i}\right):=r\left(v_{i}\right)$ for $i=1, \ldots, N$.

Output(0): The collection of radii $\left\{r_{0}\left(v_{i}\right)\right\}_{i=1}^{N}$.

Suppose that $\operatorname{Loop}(n)$ has been defined and produced the output $\left\{r_{n}\left(v_{i}\right)\right\}_{i=1}^{N}$. We are now going to describe $\operatorname{Loop}(n+1)$. 
$\operatorname{Loop}(n+1)$ : Start with the vertex $v_{1}$. Since $\mathfrak{B}$ is a branch structure for $\mathbf{T}, v_{1}$ has at least $1+\theta\left(v_{1}\right) / \pi$ neighboring vertices. Thus, we can adjust, if necessary, $r_{n}\left(v_{1}\right)$ to a value $r_{n+1}\left(v_{1}\right)$ such that the angle sum at $v_{1}$ determined by $\left\{r_{n+1}\left(v_{1}\right), r_{n}\left(v_{2}\right), \ldots, r_{n}\left(v_{N}\right)\right\} \cup\left\{R\left(w_{i}\right)\right\}_{i=1}^{M}$ is equal to $\theta\left(v_{1}\right)$.

Next, we consider the vertex $v_{2}$. Since $\mathfrak{B}$ is a branch structure for $\mathbf{T}$, $v_{2}$ has at least $1+\theta\left(v_{2}\right) / \pi$ neighboring vertices. Therefore, we can adjust, if necessary, $r_{n}\left(v_{2}\right)$ to a value $r_{n+1}\left(v_{2}\right)$ such that the angle sum at $v_{2}$ determined by $\left\{r_{n+1}\left(v_{1}\right), r_{n+1}\left(v_{2}\right), r_{n}\left(v_{3}\right), \ldots, r_{n}\left(v_{N}\right)\right\} \cup\left\{R\left(w_{i}\right)\right\}_{i=1}^{M}$ is equal to $\theta\left(v_{2}\right)$.

We continue doing these adjustments to and including $v_{N}$.

$\operatorname{Output}(n+1)$ : The collection of radii $\left\{r_{n+1}\left(v_{i}\right)\right\}_{i=1}^{N}$.

Finally, we complete the description of the algorithm by defining the final output as the collection of radii $\left\{\mathscr{R}\left(v_{i}\right)\right\}_{i=1}^{N}$, where $\mathscr{R}\left(v_{i}\right)=\liminf _{n \rightarrow \infty} r_{n}\left(v_{i}\right)$.

In what will follow we shall show that the collection of radii $\left\{\mathscr{R}\left(v_{i}\right)\right\}_{i=1}^{N} \cup$ $\left\{R\left(w_{i}\right)\right\}_{i=1}^{M}$ determines the desired branched circle packing.

We need to make a few comments about the loops and their outputs. First, unless $\mathbf{T}$ has only one interior vertex, the output of $\operatorname{Loop}(n+1)$ is never equal to the output of $\operatorname{Loop}(n)$. In the case when $\mathbf{T}$ has only one interior vertex, $\operatorname{Output}(n)=\operatorname{Output}(1)$ for any $n>1$.

Second, if $\Phi_{n}\left(v_{i}\right)$ denotes the angle sum at the vertex $v_{i}$ determined by the collection of radii $\left\{r_{n}\left(v_{1}\right), \ldots, r_{n}\left(v_{i}\right), r_{n-1}\left(v_{i+1}\right), \ldots, r_{n-1}\left(v_{N}\right)\right\} \cup\left\{R\left(w_{i}\right)\right\}_{i=1}^{M}$ then $\Phi_{n}\left(v_{i}\right)=\theta\left(v_{i}\right)$.

Third, since $\Phi\left(v_{i}\right) \leq \theta\left(v_{i}\right)$ for $i=1, \ldots, N$, the monotonicity features of angle sums imply the following two properties:

(1) $\left\{r_{n}(v)\right\}_{n=1}^{\infty}$ is a non-increasing sequence of positive numbers for every $v \in$ int $\mathbf{T}$,

(2) if $\theta_{n}(v)$ denotes the angle sum at $v$ given by $\left\{r_{n}\left(v_{i}\right)\right\}_{i=1}^{N} \cup\left\{R\left(w_{i}\right)\right\}_{i=1}^{M}$ then $\theta_{n}(v) \leq \theta(v)$ for any $n$ and $v \in$ int $\mathbf{T}$.

From property (1) we get that $\lim _{n \rightarrow \infty} r_{n}(v)$ exists for any vertex $v$, so $\mathscr{R}(v)=$ $\lim _{n \rightarrow \infty} r_{n}(v)$, though a priori $\mathscr{R}(v)$ may be zero. We also observe

Proposition 3. If $\mathscr{R}(v)>0$ then $\left\{\theta_{n}(v)\right\}_{n=1}^{\infty}$ converges to $\theta(v)$ (even if $\mathscr{R}\left(v_{j}\right)$ $=0$ for some (or all) neighboring vertices $v_{j}$ of $v$ ).

Proof. Let $u_{1}, u_{2}, \ldots, u_{l}$ be all neighboring vertices of $v$ in $\mathbf{T}$ listed in the positive order as the boundary vertices of the star of $v$ in $\mathbf{T}$. Recall that if $\rho: V(\mathbf{T}) \rightarrow(0, \infty)$ then $\theta_{\rho}(v)$ denotes the angle sum at $v$ determined by $\rho$. It follows easily from the definition of $\theta_{\rho}(\cdot)$ that $\theta_{\rho}(v)$ depends only on the values of $\rho$ at $v$ and its neighbors $u_{1}, u_{2}, \ldots, u_{l}$. More precisely, $\theta_{\rho}(v)=\theta_{\rho}\left(\rho\left(u_{1}\right), \ldots, \rho\left(u_{l}\right) ; \rho(v)\right)$, where

$$
\begin{aligned}
& \theta_{\rho}\left(\rho\left(u_{1}\right), \ldots, \rho\left(u_{l}\right) ; \rho(v)\right) \\
& =\sum_{j=1 \bmod l}^{l} \arccos \left[\frac{\left(\rho(v)+\rho\left(u_{j}\right)\right)^{2}+\left(\rho(v)+\rho\left(u_{j+1}\right)\right)^{2}-\left(\rho\left(u_{j}\right)+\rho\left(u_{j+1}\right)\right)^{2}}{2\left(\rho(v)+\rho\left(u_{j}\right)\right)\left(\rho(v)+\rho\left(u_{j+1}\right)\right)}\right] .
\end{aligned}
$$

From this formula we see that for any $\delta>0, \theta_{\rho}(v)$ is continuous as a function of $\rho$ for $\rho$ in $\underbrace{[0, \infty) \times \cdots \times[0, \infty)}_{l \text { times }} \times[\delta, \infty)$, and is uniformly continuous in any compact subset of the above product.

Let $\lambda:=\max \left\{r\left(v_{j}\right), R\left(w_{j}\right): v_{j}, w_{j} \in\left\{u_{1}, \ldots, u_{l}\right\}\right\}$. We observe that each argument of the angle function $\theta_{n}(v)$ is at least as big as the corresponding 
argument of the angle function $\Phi_{n+1}(v)$ but not bigger than the corresponding argument of $\Phi_{n}(v)$. Thus, if $\mathscr{R}(v)>0$ then $\theta_{\rho}(v)$ is uniformly continuous for $\rho$ in $[0, \lambda]^{l} \times[\mathscr{R}(v) / 2, r(v)]$ and, by property (1), one has

$$
\theta(v) \equiv \Phi_{n}(v)=\lim _{n \rightarrow \infty} \Phi_{n}(v)=\lim _{n \rightarrow \infty} \theta_{n}(v)
$$

that is, the limit of $\left\{\theta_{n}(v)\right\}_{n=1}^{\infty}$ exists and is equal to $\theta(v)$.

Since $\mathscr{R}(v)$ may be zero, we have to consider two cases.

Case 1. $\mathscr{R}(v)>0$ for all $v \in$ int $\mathbf{T}$.

It follows from Proposition 3 that $\left\{\theta_{n}\left(v_{i}\right)\right\}_{i=1}^{\infty}$ converges to $\theta\left(v_{i}\right)$ for any $i$, and, as the $\theta\left(v_{i}\right)$ are multiples of $2 \pi$, the collection of radii $\left\{\mathscr{R}\left(v_{i}\right)\right\}_{i=1}^{N} \cup$ $\left\{R\left(w_{i}\right)\right\}_{i=1}^{M}$ defines the sought after branched circle packing.

Case 2. There exists $i_{0}, 1 \leq i_{0} \leq N$, such that $\mathscr{R}\left(v_{i_{0}}\right)=0$.

We will show that this case is void.

We extend the definition of $r_{n}(\cdot)$ and $\mathscr{R}(\cdot)$ to the boundary vertices of $\mathbf{T}$ by $r_{n}\left(w_{i}\right):=R\left(w_{i}\right)$ and $\mathscr{R}\left(w_{i}\right):=\lim _{n \rightarrow \infty} r_{n}\left(w_{i}\right)=R\left(w_{i}\right)$. Now let us call a vertex $u$ a solid dot if $\mathscr{R}(u)>0$ and an open dot if $\mathscr{R}(u)=0$. Let $O D$ be the collection of all open dots and $S D$ be the collection of all solid dots. Notice that all boundary vertices $w_{i}$ are solid dots. Hence $S D \neq \varnothing$. Also, by the hypothesis, $O D \neq \varnothing$ (because $v_{i_{0}} \in O D$ ).

We now need to introduce some technical notation which otherwise is quite natural. If $X$ is a subcomplex of $\mathbf{T}$ (not necessarily a 2-complex) then $V(X)$ will denote the set of vertices of $X$. We will call $v \in V(X)$ an interior vertex of $X$ if $v$ is an interior vertex of $\mathbf{T}$ and all neighboring vertices of $v$ in $\mathbf{T}$ belong to $V(X)$. If $v \in V(X)$ is not interior then it will be called a boundary vertex of $X$. The sets of interior and boundary vertices of $X$ will be denoted by int $X$ and bd $X$, respectively. If $\gamma$ is an edge-path in $\mathbf{T}$ and $X \subset V(\mathbf{T})$ then we will say that $\gamma$ is in $X$ if $V(\gamma) \subset X$.

If $\gamma$ is a closed oriented edge-path in $\mathbf{T}$, possibly with self-intersections, then the set $\left\{u_{1}, \ldots, u_{l}\right\}$ of consecutive vertices of $\gamma$ will be denoted by $V_{c}(\gamma)$ and $u_{j+1}$ will be called the successor of $u_{j}$ ( $j$ mod $l$, of course). We observe that $V\left(V_{c}(\gamma)\right)=V(\gamma) \subseteq V_{c}(\gamma)$ with equality if and only if $\gamma$ is a simple closed path (i.e., $V_{c}(\gamma)$ has exactly the same elements as $V(\gamma)$ but they may appear with repetitions in $V_{c}(\gamma)$ if $\gamma$ has self-intersections). Finally, $\mathbf{u}_{\mathbf{j}-1} \mathbf{u}_{\mathbf{j}} \mathbf{u}_{\mathbf{j}+1}$ will denote an oriented path given by the part of $\gamma$ between $u_{j-1}$ and $u_{j+1}$ that contains $u_{j}$.

A subset $X \subset V(\mathbf{T})$ is called connected if any two vertices in $X$ can be joined by an edge-path in $X$; it is simply connected if there is no closed edge-path in $X$ which encloses an element from $V(\mathbf{T}) \backslash X$. If $X \subset V(\mathbf{T})$ then $\operatorname{hull}(X)$ will denote the combinatorial hull of $X$, i.e., the union of the set $X$ and the collection of all vertices in $V(\mathbf{T})$ such that each vertex (from the collection) is enclosed by some edge-path in $X$.

The next few definitions are only for subsets of int T. If $X \subset$ int $\mathbf{T}$ then the outer boundary out $X$ of $X$ is the set of all vertices in $\mathbf{T}$ which are not in $\operatorname{hull}(X)$ but which have a neighboring vertex in hull $(X)$. A subset $X \subset$ int $\mathbf{T}$ is said to be bounded by an edge-path $\gamma$ (or $\gamma$ bounds $X$ ) if $\operatorname{hull}(X)$ is connected and $\gamma$ is the boundary curve of the union $U(X)$ of open stars associated with 
the vertices of $\operatorname{hull}(X)$; in such case, $\gamma$ is closed and carries an orientation induced by the orientation of the set $U(X)$ (with respect to which $U(X)$ stays to the left of $\gamma)$. We notice that any subset $X \subset$ int T with connected hull $(X)$ is bounded by some edge-path that in fact encloses $X$ and, in general, such a path may have vertices or even edges of self-tangency (but not transverse). If $X$ is bounded by a path $\gamma$ then we will write $\partial_{e} X$ for $\gamma$ and $V_{c}(X)$ for $V_{c}\left(\partial_{e} X\right)$, and we observe that $V\left(\partial_{e} X\right)=$ out $X$. If a subset $X \subset$ int $\mathbf{T}$ is simply connected then $(\operatorname{bd}(V(\mathbf{T}) \backslash X) \backslash \mathrm{bd} \mathbf{T}) \cup(\mathrm{bd} \mathbf{T} \cap$ out $X)=$ out $X$.

Let $C_{O D}$ and $C_{S D}$ denote components of $O D$ and $S D$, respectively. We say that an edge-path $\gamma$ in $\mathbf{T}$ is a path of open (solid) dots if $V(\gamma) \subset O D$ $(V(\gamma) \subset S D)$. We also remark that $C_{O D}\left(C_{S D}\right)$ is simply connected if and only if there is no closed edge-path in $C_{O D}\left(C_{S D}\right)$ that encloses at least one point from $O D(S D)$.

We will now state a lemma; only the first four parts, (i)-(iv), will be needed to prove Theorem 2, the other parts, (v)-(vii), are stated for completeness and future applications. We want to remark that this lemma is the key element in our proof of Theorem 2, represents an independent approach in comparison with the methods used in [B] and [G], and plays a crucial role in a proof of the existence of infinite branched circle packings [D].

Lemma 4. Under the assumption that $\left\{\left(b_{1}, k_{1}\right), \ldots,\left(b_{m}, k_{m}\right)\right\}$ is a branch structure for the finite triangulation $\mathrm{T}$ and that sets $O D$ and $S D$ are defined for the function $\mathscr{R}$ as above, the following hold:

(i) There is no simply connected component $C_{O D}$ of $O D$.

(ii) There is no simply connected component $C_{S D}$ of $S D$ except for $V(\mathbf{T})$.

(iii) There is no subset $S \subset$ int $\mathbf{T}$ that contains at least one solid dot and is bounded by a path of open dots.

(iv) There is no subset $S \subset$ int $\mathbf{T}$ that contains at least one open dot and is bounded by a path of solid dots.

(v) There is no subset $S \subset S D \cap$ int $\mathbf{T}$ so that $S$ is simply connected and out $S$ consists only of open dots except one solid dot.

(vi) There is no connected subset $S$ of $S D \cap \operatorname{int} \mathbf{T}$ with out $S$ having only one solid dot.

(vii) There is no connected subset $S$ in int $\mathbf{T}$ so that $S$ contains at least one solid dot and out $S$ has only one solid dot.

Proof. (i) Suppose $C_{O D}$ is a simply connected component of $O D$. Since bd T $\subset S D, C_{O D} \subset$ int T and $\partial_{e} C_{O D}$ is in $S D$ (Figure 1).

For three consecutive vertices $u_{j-1}, u_{j}, u_{j+1}$ on $\partial_{e} C_{O D}$ let $S_{j}$ denote the part of the star of $u_{j}$ that stays to the left of the path $u_{j-1} u_{j} u_{j+1}$ (Figure 2).

Let $r_{n}: V(\mathbf{T}) \rightarrow(0, \infty)$ be defined as above, and let $\theta_{n}(\cdot), \alpha_{n}(\cdot, \cdot)$ denote $\theta_{r_{n}}(\cdot), \alpha_{r_{n}}(\cdot, \cdot)$, respectively (see the notation for $\left.(\mathrm{GB})\right)$. Let $\gamma_{n}\left(u_{j}\right):=$ $\sum_{\triangle \in S_{j}} \alpha_{n}\left(u_{j}, \triangle\right)$, i.e., $\gamma_{n}\left(u_{j}\right)$ is the interior angle with respect to $u_{j-1} u_{j} u_{j+1} \subset$ $\partial_{e} C_{O D}$ at $u_{j} \in V_{c}\left(C_{O D}\right)$ determined by $r_{n}(\cdot)$. Since each triangle $\triangle \in S_{j}$ has a vertex in $C_{O D}$, we have $\lim _{n \rightarrow \infty} \gamma_{n}\left(u_{j}\right)=0$. Also, by $(2), \theta_{n}\left(v_{i}\right) \leq \theta\left(v_{i}\right)$ for any $v_{i} \in C_{O D}$. By $(G B)$

$$
\sum_{v_{i} \in C_{O D}}\left(2 \pi-\theta_{n}\left(v_{i}\right)\right)=2 \pi-\sum_{u_{j} \in V_{c}\left(C_{O D}\right)}\left(\pi-\gamma_{n}\left(u_{j}\right)\right) .
$$

Let $b_{i_{1}}, \ldots, b_{i_{L}}$ be all $b_{i}$ 's that are in $C_{O D}$. The hypothesis (i.e., $\left\{\left(b_{1}, k_{1}\right), \ldots\right.$, 


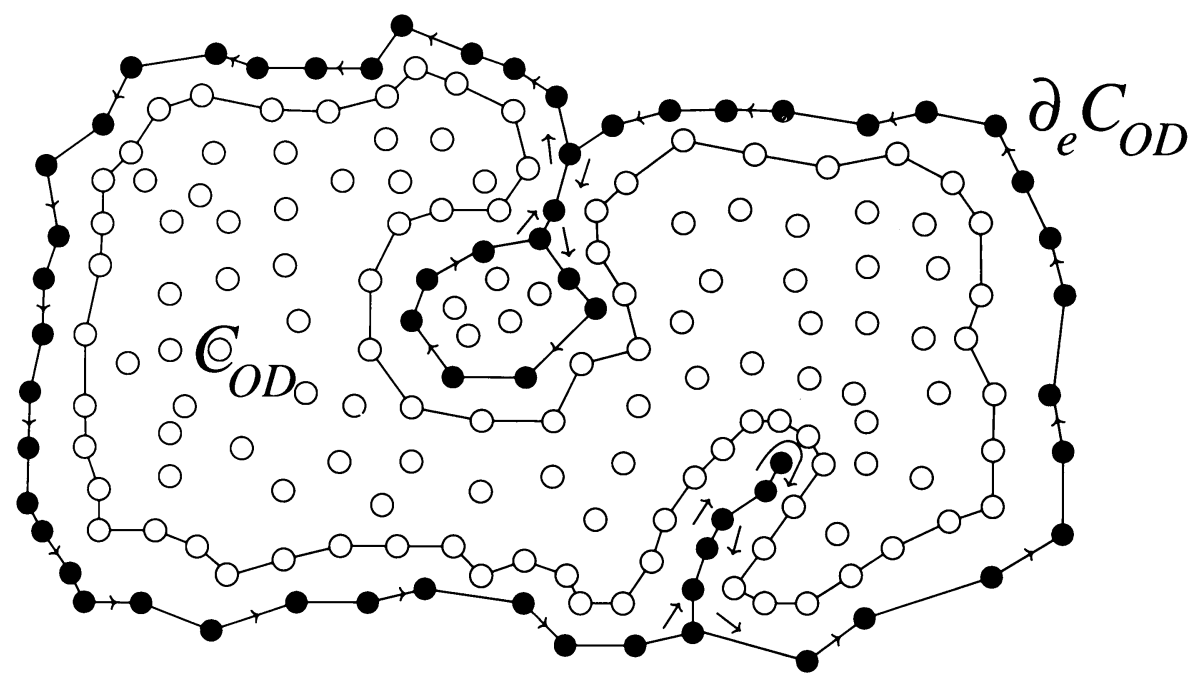

Figure 1. A set $C_{O D}$ and a path $\partial_{e} C_{O D}$ with its orientation indicated by arrows.

$\left.\left(b_{m}, k_{m}\right)\right\}$ is a branch structure for $\left.\mathbf{T}\right)$ implies that the number of $u_{j}$ 's in $V_{c}\left(C_{O D}\right)$ is at least $2\left(\sum_{j=1}^{L} k_{i_{j}}\right)+3$. Thus

$(* *)_{1}$

$$
\lim _{n \rightarrow \infty}\left[2 \pi-\sum_{u_{j} \in V_{c}\left(C_{O D}\right)}\left(\pi-\gamma_{n}\left(u_{j}\right)\right)\right]=2 \pi-\sum_{u_{j} \in V_{c}\left(C_{O D}\right)} \pi
$$

$$
\leq 2 \pi-\left[2\left(\sum_{j=1}^{L} k_{i_{j}}\right)+3\right] \pi=-\pi-2\left(\sum_{j=1}^{L} k_{i_{j}}\right) \pi .
$$

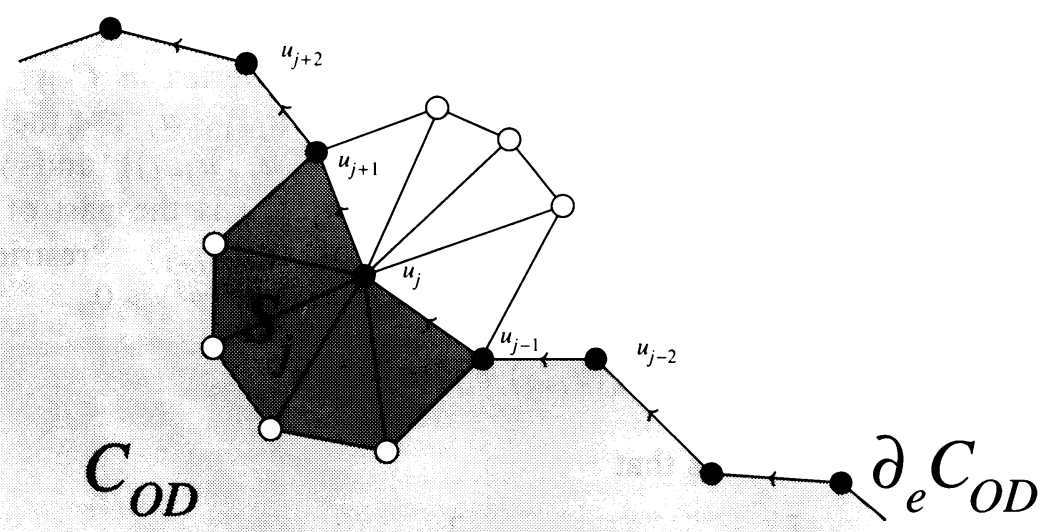

Figure 2. The set $S_{j}$ for $C_{O D}$. 


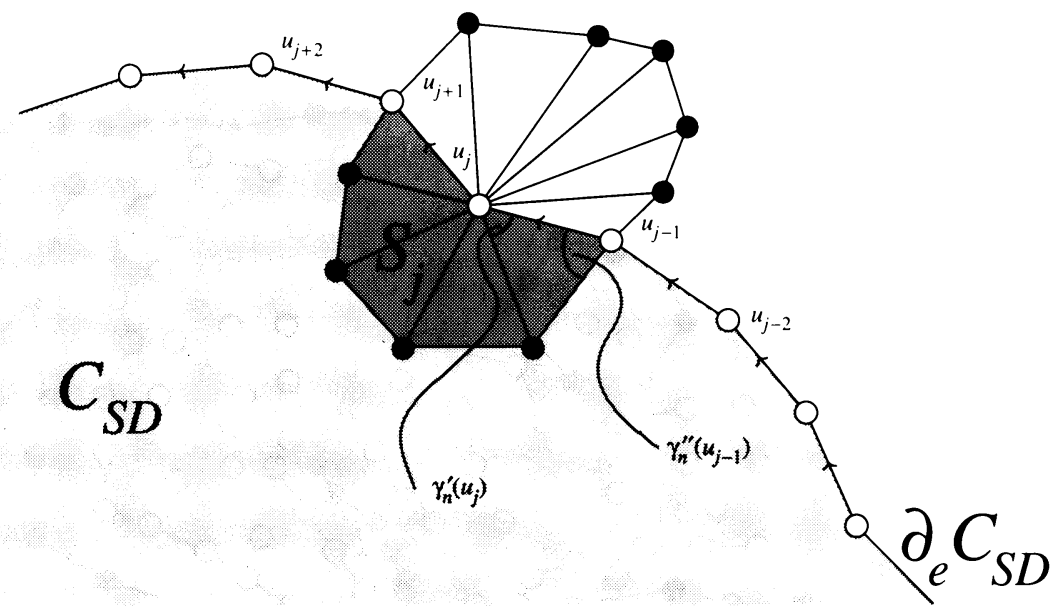

Figure 3. The set $S_{j}$ for $C_{S D}$.

On the other hand

$$
(* *)_{2} \sum_{v_{i} \in C_{O D}}\left(2 \pi-\theta_{n}\left(v_{i}\right)\right) \geq \sum_{v_{i} \in C_{O D}}\left(2 \pi-\theta\left(v_{i}\right)\right)=\sum_{j=1}^{L}\left(2 \pi-\theta\left(b_{i_{j}}\right)\right)=-\sum_{j=1}^{L} 2 k_{i_{j}} \pi
$$

for any $n$. Comparing $(* *)_{1}$ and $(* *)_{2}$ one obtains a contradiction.

(ii) Suppose there exists $C_{S D} \neq V(\mathbf{T})$ which is simply connected. Then bd $\mathbf{T} \cap C_{S D}=\varnothing$, for otherwise the edge-path of boundary edges of $\mathbf{T}$ is a closed path in $C_{S D}$ that encloses some $v_{i_{0}} \in O D$, implying that $C_{S D}$ not simply connected. Hence $C_{S D} \subset$ int $\mathrm{T}$ and $\partial_{e} C_{S D}$ is in $O D$.

For three consecutive (open) dots $u_{j-1}, u_{j}, u_{j+1}$ on $\partial_{e} C_{S D}$ let $S_{j}$ denote the part of the star of $u_{j}$ that stays to the left of the path $u_{j-1} u_{j} u_{j+1}$ (Figure 3 ).

Let $\gamma_{n}\left(u_{j}\right):=\sum_{\Delta \in S_{j}} \alpha_{n}\left(u_{j}, \triangle\right)$ (notation as in the proof of Part (i)), i.e., $\gamma_{n}\left(u_{j}\right)$ is the interior angle with respect to $u_{j-1} u_{j} u_{j+1} \subset \partial_{e} C_{S D}$ at $u_{j} \in V_{c}\left(C_{S D}\right)$ determined by $r_{n}(\cdot)$. Since each triangle in $S_{j}$ has a vertex in $C_{S D}$, for each $u_{j} \in \partial_{e} C_{S D}$ there exists $v^{j} \in C_{S D}$ such that $v^{j}, u_{j-1}, u_{j}$ are the vertices of a triangle in $S_{j}$. Let $\gamma_{n}^{\prime}\left(u_{j}\right):=\alpha_{n}\left(u_{j}, \triangle\left(v^{j}, u_{j}, u_{j-1}\right)\right)$ and $\gamma_{n}^{\prime \prime}\left(u_{j-1}\right)$ $:=\alpha_{n}\left(u_{j-1}, \Delta\left(v^{j}, u_{j}, u_{j-1}\right)\right)$, i.e., $\gamma_{n}^{\prime}\left(u_{j}\right)\left(\gamma_{n}^{\prime \prime}\left(u_{j-1}\right)\right)$ is the part of $\gamma_{n}\left(u_{j}\right)$ "restricted to $\triangle\left(v^{j}, u_{j}, u_{j-1}\right)$ " (the part of $\gamma_{n}\left(u_{j-1}\right)$ "restricted to $\left.\triangle\left(v^{j}, u_{j}, u_{j-1}\right) "\right)$. Since $\mathscr{R}\left(u_{j-1}\right)=\mathscr{R}\left(u_{j}\right)=0$ and $\mathscr{R}\left(v^{j}\right)>0$,

$$
\lim _{n \rightarrow \infty}\left(\gamma_{n}^{\prime \prime}\left(u_{j-1}\right)+\gamma_{n}^{\prime}\left(u_{j}\right)\right)=\pi
$$

From this remark it follows that

$$
(* * *) \quad \lim _{n \rightarrow \infty}\left[\sum_{u_{j} \in V_{c}\left(C_{S D}\right)}\left(\pi-\gamma_{n}\left(u_{j}\right)\right)\right]=A \text {, where } A \in(-\infty, 0] .
$$

Now, if $v_{i} \in C_{S D}$ then $\lim _{n \rightarrow \infty} \theta_{n}\left(v_{i}\right)=\theta\left(v_{i}\right)$ by Proposition 3 . 
Since $\theta\left(v_{i}\right) \geq 2 \pi$, we have

$$
(* * *)_{1} \quad \lim _{n \rightarrow \infty}\left[\sum_{v_{i} \in C_{S D}}\left(2 \pi-\theta_{n}\left(v_{i}\right)\right)\right]=-\sum_{j=1}^{L} 2 k_{i_{j}} \pi,
$$

where $b_{i_{1}}, \ldots, b_{i_{L}}$ are all $b_{i}$ 's that are in $C_{S D}$. By (GB)

$$
\begin{aligned}
0 & \geq-\sum_{j=1}^{L} 2 k_{i_{j}} \pi=\lim _{n \rightarrow \infty}\left[\sum_{v_{i} \in C_{S D}}\left(2 \pi-\theta_{n}\left(v_{i}\right)\right)\right] \\
& =\lim _{n \rightarrow \infty}\left[2 \pi-\sum_{u_{j} \in V_{c}\left(C_{S D}\right)}\left(\pi-\gamma_{n}\left(u_{j}\right)\right)\right]=2 \pi-A \geq 2 \pi,
\end{aligned}
$$

and one gets a contradiction.

(iii) Suppose there is such a subset $S$. Let $S_{S D}$ be a component in $\operatorname{hull}(S)$ of solid dots. We notice that $S_{S D}$ is one of the $C_{S D}$ 's because out $S=$ $\operatorname{out}(\operatorname{hull}(S)) \subset O D$. By Part (ii), $S_{S D}$ is not simply connected. Hence there is a path in $S_{S D}$ that encloses at least one open dot in hull $(S)$. Let $S_{O D}$ be the component in hull $(S)$ of open dots that contains the above open dot. Since $S_{O D}$ is enclosed by a path in $S_{S D}, S_{O D}$ is one of the $C_{O D}$ 's, and, by Part (i), is not simply connected. We now apply to $S_{O D}$ the same type of argument as we did above to $S_{S D}$, and by continuing this process we will eventually end up with a simply connected component of one type of dots. The last yields a contradiction to Part (i) or (ii).

(iv) This part follows from the same arguments that were used in the proof of Part (iii).

(v) Suppose that $S$ exists. Denote the only solid vertex of out $S$ by $u^{\prime}$. Then $u^{\prime}$ can not separate out $S$, i.e., out $S \backslash\left\{u^{\prime}\right\}$ must be connected. For if $u^{\prime}$ separates out $S$ then there is a closed path of solid dots (including $u^{\prime}$ ) that bounds a region in int $\mathbf{T}$ which contains one of the components of out $S \backslash\left\{u^{\prime}\right\}$. But by Part (iv) that is impossible.

Hence $u^{\prime}$ does not separate out $S$ and therefore there exists unique $u_{j_{0}} \in$ $V_{c}(S)$ such that $u^{\prime}=u_{j_{0}}$, i.e., $u_{j_{0}}$ is the only solid dot in $V_{c}(S)$. Using the notation as in the proof of Part (ii), we easily obtain from the definition of solid and open dots that $\lim _{n \rightarrow \infty} \gamma_{n}^{\prime \prime}\left(u_{j_{0}-1}\right)=\pi=\lim _{n \rightarrow \infty} \gamma_{n}^{\prime}\left(u_{j_{0}+1}\right)$. Applying the arguments from the proof of Part (ii), the above observation, and the fact that $u_{j_{0}}$ is the only solid dot in $V_{c}(S)$, we conclude that

$$
\lim _{n \rightarrow \infty}\left[\sum_{u_{j} \in V_{c}(S) \backslash\left\{u_{j_{0}}\right\}}\left(\pi-\gamma_{n}\left(u_{j}\right)\right)\right]=C \text {, where } C \in(-\infty, 0] \text {. }
$$

Since $\gamma_{n}\left(u_{j_{0}}\right) \geq 0$, from (1) we have

$$
\sum_{u_{j} \in V_{c}(S)}\left(\pi-\gamma_{n}\left(u_{j}\right)\right) \leq 3 / 2 \pi \text { for large } n .
$$

As in the proof of Part (ii)

$$
\lim _{n \rightarrow \infty}\left[\sum_{v_{i} \in S}\left(2 \pi-\theta_{n}\left(v_{i}\right)\right)\right]=-\sum_{j=1}^{L} 2 k_{i_{j}} \pi
$$


where $b_{i_{1}}, \ldots, b_{i_{L}}$ are all $b_{i}$ 's that are in $S$. Using (2), (3) and $(G B)$ $0 \geq \sum_{v_{i} \in S}\left(2 \pi-\theta_{n}\left(v_{i}\right)\right)=2 \pi-\sum_{u_{j} \in V_{c}(S)}\left(\pi-\gamma_{n}\left(u_{j}\right)\right) \geq \pi / 2$ for sufficiently large $n$, but this is a contradiction.

(vi) Suppose that $S$ exists. Then, by Part (v), $S$ can not be simply connected, and we have only two possibilities. The first one is that there exists a closed edge-path in $S \subset S D$ that encloses an element from $O D$, but this contradicts Part (iv). Hence we are left with the second possibility which is: hull $(S) \subset$ $S D$. Since $\operatorname{hull}(S)$ is simply connected and out $S=\operatorname{out}($ hull $(S))$, we get a contradiction to Part (v).

(vii) Suppose that $S$ exists. Take the component $C$ of solid dots in $S \cup$ out $S$ that contains the only boundary solid dot $u^{\prime} \in$ out $S$. If $C=\left\{u^{\prime}\right\}$ then there is a region which is bounded by a path of open dots in out $\left(S \cup\left(\right.\right.$ out $\left.\left.S \backslash\left\{u^{\prime}\right\}\right)\right) \subset$ $S \cup$ out $S$ and which contains at least one solid dot from $S$. But this contradicts Part (iii).

If $C \neq\left\{u^{\prime}\right\}$ then Part (vi) applies to $C \backslash\left\{u^{\prime}\right\}$, and we again get a contradiction.

We can now finish the proof of Theorem 2 .

Part (iv) of Lemma 4 says that our assumption that $\mathscr{R}\left(v_{i_{0}}\right)=0$ is false. Hence we are in Case 1 and one implication in our theorem is proved, i.e., the existence.

The other implication (i.e., the necessity) follows easily from (GB), and this completes the proof of Theorem 2.

We will now state a theorem that addresses the boundary value problem for branched circle packings in $\mathbf{R}^{2}$ and in the hyperbolic plane; i.e., having been given geometry and prescribed radii for boundary vertices, can one construct a packing in the given geometry with boundary circles having those prescribed radii?

Theorem 5. Let $\mathbf{T}$ be a finite triangulation of a disc. Suppose $\mathfrak{B}=\left\{\left(b_{1}, k_{1}\right)\right.$, $\left.\ldots,\left(b_{m}, k_{m}\right)\right\}$ is a branch structure for $\mathbf{T}$. Then, in either euclidean or hyperbolic geometry, there exists a branched circle packing determined by $\mathbf{T}$ and $\mathfrak{B}$ with any prescribed boundary radii. Moreover, such a packing is unique up to isometries in the corresponding geometry.

Proof. Euclidean case. Let $\widetilde{P}$ be a locally univalent circle packing for $\mathbf{T}$ that has prescribed boundary radii. Such a packing exists by [CR]. We now replace $A$ in the proof of Theorem 2 by $\widetilde{P}$, and we follow the method of construction of a branched circle packing in that proof. We observe that this construction does not change the boundary radii of $\widetilde{P}$. Hence we obtain the desired branched circle packing with prescribed boundary radii.

Regarding the uniqueness, suppose $P_{1}$ and $P_{2}$ are two branched circle packings determined by $\mathbf{T}$ and $\mathfrak{B}$ having the same boundary radii. Let $\mathscr{R}_{i}(\cdot)$ : $V(\mathbf{T}) \rightarrow(0, \infty)$ be the radius function for $P_{i}$, and define $\frac{\mathscr{R}_{1}}{\mathscr{R}_{2}}(\cdot): V(\mathbf{T}) \rightarrow$ $(0, \infty)$ by $\frac{\mathscr{R}_{1}}{\mathscr{R}_{2}}(u)=\mathscr{R}_{1}(u) / \mathscr{R}_{2}(u)$. Since $P_{1}$ and $P_{2}$ have the same angle sums at corresponding vertices, one easily verifies that $\frac{\mathscr{R}_{1}}{\mathscr{R}_{2}}(\cdot)$ satisfies the maximum 
and minimum principle, i.e., $\frac{\mathscr{R}_{1}}{\mathscr{R}_{2}}(\cdot)$ cannot attain its maximum or minimum in int $\mathbf{T}$ unless it is a constant function. This implies that $\mathscr{R}_{1}(\cdot) \equiv \mathscr{R}_{2}(\cdot)$, as $\frac{\mathscr{R}_{1}}{\mathscr{R}_{2}}(\cdot)$ restricted to bd $\mathrm{T}$ is equal to 1 .

Hyperbolic case. Theorem 2 gives the existence of a branched circle packing determined by $\mathbf{T}$ and $\mathfrak{B}$ in the hyperbolic plane (one just takes any euclidean branched circle packing determined by $\mathbf{T}$ and $\mathfrak{B}$, scales it down so it will be contained in the unit disc). Using this information together with some straightforward generalizations of the techniques used in [BSt2] for the locally univalent setting, like the Perron method, one easily obtains the conclusion of the theorem in the hyperbolic setting.

We finish this section with

Definition 6. Let $\mathbf{T}$ be a finite triangulation of a disc. Suppose $\mathfrak{B}$ is a branch structure for $\mathbf{T}$. Let $P$ be a branched circle packing in the unit disc determined by $\mathbf{T}$ and $\mathfrak{B}$ with all boundary circles being horocycles. Then $P$ will be called a Bl-type packing determined by $\mathbf{T}$ and $\mathfrak{B}$.

\section{EXTENSIONS OF SIMPLICIAL MAPS}

Suppose that $\mathfrak{B}=\left\{\left(b_{1}, k_{1}\right), \ldots,\left(b_{m}, k_{m}\right)\right\}$ is a branch structure for a finite triangulation $\mathbf{T}$ of a disc. Let $A$ be an (orientation preserving) Andreev packing for $\mathbf{T}$, and let $B$ be a (orientation preserving) Bl-type packing determined by $\mathbf{T}$ and $\mathfrak{B}$. Write $f$ for the simplicial map from $A$ to $B$. Our goal in this section is to show that one can think about $f$ as a discrete Blaschke product.

As in the previous section, let $\left\{w_{i}\right\}$ denote the set of boundary vertices of T. Assume that the $w_{i}$ 's are indexed so that they induce counterclockwise orientation of the boundary of $\mathbf{T}$. For any two points $p$ and $q$ in the unit circle $\partial D,\langle p, q$ l will represent the oriented arc of $\partial D$ from $p$ to $q$, where the orientation of $\partial D$ is counterclockwise. Also, for any two points $p, q \in \mathbf{C}$, we will write $[p, q]$ for the segment in $\mathbf{C}$ with the ends $p$ and $q$. For a vertex $u \in V(\mathbf{T}), C_{A}(u)$ and $C_{B}(u)$ will denote the circles that correspond to $u$ in $A$ and $B$, respectively; $\widehat{u}$ will denote the center of $C_{A}(u)$, so that $f(\widehat{u})$ is the center of $C_{B}(u)$.

Suppose that $a, b$, and $c$ are the points of tangency of $C_{B}\left(w_{2}\right)$ with $\partial D$, $C_{B}\left(w_{3}\right)$, and $C_{B}\left(w_{1}\right)$, respectively. Let all circles in $A$ and $B$ have counterclockwise orientation. Since $B$ is orientation preserving one has

Remark 1. The ordered triple $\langle a, b, c\rangle$ has the order that agrees with the orientation of $C_{B}\left(w_{2}\right)$, i.e., starting at $a$ and going along the circle $C_{B}\left(w_{2}\right)$ according to its orientation first we encounter $b$ then $c$.

Remark 2. Let $\bar{w}_{i}^{\prime}=\partial D \cap C_{B}\left(w_{i}\right)$. Then, using Remark 1 , one easily verifies that $\left\langle\bar{w}_{i}^{\prime}, \bar{w}_{i+1}^{\prime}, \bar{w}_{i+2}^{\prime}\right\rangle$ has the order that agrees with the orientation of $\partial D$.

We are now going to build an extension of $f$ to a map from $\bar{D}$ onto $\bar{D}$ as follows:

Let $\bar{w}_{i}=\partial D \cap C_{A}\left(w_{i}\right)$. In each arc $\left\{\bar{w}_{i}, \bar{w}_{i+1}\right\rceil \subset \partial D$ pick a point $x_{i}$ such that $\left[\widehat{w}_{i}, x_{i}\right] \cap C_{A}\left(w_{i+1}\right)=\varnothing=\left[\widehat{w}_{i+1}, x_{i}\right] \cap C_{A}\left(w_{i}\right)$. Similarly, in every arc $\left\langle\bar{w}_{i}^{\prime}, \bar{w}_{i+1}^{\prime} \backslash \subset \partial D\right.$ pick a point $y_{i}$ such that $\left[f\left(\widehat{w}_{i}\right), y_{i}\right] \cap C_{B}\left(w_{i+1}\right)=\varnothing=\left[f\left(\widehat{w}_{i+1}\right), x_{i}\right] \cap C_{B}\left(w_{i}\right)$. Then we extend our map $f$ in the following way: 
(1) Each closed triangle $\Delta x_{i} \widehat{w}_{i} \widehat{w}_{i+1}$ is mapped simplicially onto the closed triangle $\Delta y_{i} f\left(\widehat{w}_{i}\right) f\left(\widehat{w}_{i+1}\right)$ by mapping $x_{i}, \widehat{w}_{i}, \widehat{w}_{i+1}$ onto $y_{i}, f\left(\widehat{w}_{i}\right), f\left(\widehat{w}_{i+1}\right)$, respectively. Hence the extended map agrees with $f$ on each segment $\left[\widehat{w}_{i}, \widehat{w}_{i+1}\right]$, so is well defined.

(2) Next, every closed triangular region $\triangle\left(x_{i} x_{i+1} \widehat{w}_{i+1}\right)$ (bounded by $\left[x_{i}, \widehat{w}_{i+1}\right],\left[x_{i+1}, \widehat{w}_{i+1}\right]$ and $\left.x_{i}, x_{i+1} l \subset \partial D\right)$ is mapped homeomorphically onto the closed triangular region $\triangle\left(y_{i} y_{i+1} f\left(\widehat{w}_{i+1}\right)\right)$ so that:

- $x_{i}, x_{i+1}, \widehat{w}_{i+1}$ are mapped onto $y_{i}, y_{i+1}, f\left(\widehat{w}_{i+1}\right)$, respectively, - the arc $l x_{i}, x_{i+1} l$ is mapped onto $l y_{i}, y_{i+1} l$, and the homeomorphism agrees on segments $\left[x_{i}, \widehat{w}_{i+1}\right],\left[x_{i+1}, \widehat{w}_{i+1}\right]$ with the extension done in step (1).

This completes the construction of an extension of $f$.

Let us call the extension defined above $\bar{f}$. Remark 2 implies that the "star" of $\widehat{w}_{i}$ (the union of: all faces in $\operatorname{carr}(A)$ having $\widehat{w}_{i}$ as a vertex, two triangles $\Delta \widehat{w}_{i-1} \widehat{w}_{i} x_{i}$ and $\Delta \widehat{w}_{i+1} \widehat{w}_{i} x_{i}$, and a triangular region $\left.\Delta\left(x_{i} x_{i+1} \widehat{w}_{i}\right)\right)$ is mapped homeomorphically by $\bar{f}$ onto the "star" of $f\left(\widehat{w}_{i}\right)$ (the union of: all faces in $\operatorname{carr}(B)$ having $f\left(\widehat{w}_{i}\right)$ as a vertex, two triangles $\Delta f\left(\widehat{w}_{i-1}\right) f\left(\widehat{w}_{i}\right) y_{i}$ and $\triangle f\left(\widehat{w}_{i+1}\right) f\left(\widehat{w}_{i}\right) y_{i}$, and the triangular region $\left.\triangle\left(y_{i} y_{i+1} f\left(\widehat{w}_{i}\right)\right)\right)$. Now it easily follows from the definition of $f$ and the construction of $\bar{f}$ that $\bar{f}: \bar{D} \rightarrow \bar{D}$ is a local homeomorphism in $\bar{D} \backslash\left\{\widehat{b}_{1}, \ldots, \widehat{b}_{m}\right\}, \bar{f}(\partial D) \subseteq \partial D$, and $\left.\bar{f}\right|_{D}$ is open and light (i.e., every component of $\bar{f}^{-1}(\xi)$ is a single point for any $\left.\xi \in \bar{f}(D)\right)$. By the Stoilow's theorem (see [LV]) we get that $\left.\bar{f}\right|_{D}=\varphi \circ h$, where $h$ is a homeomorphism of $D$ and $\varphi$ is a holomorphic function in $h(D)$. A priori $h(D)$ may or may not be equal to $\mathbf{C}$. If $h(D)=\mathbf{C}$ then $\varphi$ is an entire analytic function that is bounded (because $\bar{f}: \bar{D} \rightarrow \bar{D}$ ). Hence $\varphi$ is constant. But $\bar{f}$ is not constant, contradiction. Therefore $h(D) \neq \mathbf{C}$. Since $h(D)$ is simply connected, by the Riemann mapping theorem there exists a conformal map $g: h(D) \rightarrow D$. Thus $\left.\bar{f}\right|_{D}=\varphi_{1} \circ h_{1}$, where $h_{1}: D \rightarrow D$ is a homeomorphism and $\varphi_{1}: D \rightarrow D$ is analytic.

Let $\left\{z_{n}\right\}_{n=1}^{\infty}$ be a sequence of points from $D$ that converges to $\partial D$. Then $\left\{h_{1}^{-1}\left(z_{n}\right)\right\}$ converges to $\partial D$. Write $z_{n}^{\prime}:=h_{1}^{-1}\left(z_{n}\right)$. Since $\bar{f}$ is continuous in $\bar{D}$ and $\bar{f}(\partial D) \subseteq \partial D,\left\{\bar{f}\left(z_{n}^{\prime}\right)\right\}$ converges to $\partial D$. Hence points $\varphi_{1}\left(z_{n}\right)=\bar{f}\left(z_{n}^{\prime}\right)$ converge to $\partial D$. Therefore $\varphi_{1}$ is a proper analytic map from $D$ into $D$, so $\varphi_{1}$ is a finite Blaschke product (please refer to the characterization of Blaschke products in the next section).

All that we can summarize in

Theorem 3. Let $\bar{f}$ be an extended map from $A$ to $B$ described as above. Then $\left.\bar{f}\right|_{D}=\varphi \circ h$, where $h$ is a homeomorphism of $D$ and $\varphi$ is a finite Blaschke product having branch points $h\left(\widehat{b}_{1}\right), \ldots, h\left(\widehat{b}_{m}\right)$ with orders $k_{1}, \ldots, k_{m}$, respectively.

The above theorem justifies us in stating the following

Definition 4. Suppose that $\mathfrak{B}=\left\{\left(b_{1}, k_{1}\right), \ldots,\left(b_{m}, k_{m}\right)\right\}$ is a branch structure for a finite triangulation $\mathbf{T}$ of a disc. Let $A$ be an Andreev circle packing for $\mathbf{T}$, and let $B$ be a Bl-type circle packing determined by $\mathbf{T}$ and $\mathfrak{B}$. If $f$ is the simplicial map from $A$ to $B$ then $f$ will be called the discrete Blaschke 
product from $A$ to $B$. Moreover, a map $f$ will be called a discrete Blaschke product induced by $B$ if $f$ is the discrete Blaschke product from $A$ to $B$ for some $A$.

We finish this section with a lemma which says that overlapping petals of a flower in a Bl-type packing must trap a branch value of any discrete Blaschke product induced by the packing.

Lemma 5. Let $\mathbf{T}$ be a finite triangulation of a disk. Suppose $B$ is a Bl-type circle packing determined by $\mathbf{T}$ and the branch set $\mathfrak{B}=\left\{\left(b_{1}, k_{1}\right), \ldots,\left(b_{m}, k_{m}\right)\right\}$. Write $f$ for a discrete Blaschke product induced by $B$. Let $v_{0}$ be an interior vertex of $\mathbf{T}$. Suppose there are non-adjacent vertices $v_{1}$ and $v_{2}$, both neighboring $v_{0}$, such that $C\left(v_{1}\right) \cap C\left(v_{2}\right) \neq \varnothing$, where $C\left(v_{j}\right)$ is the circle in $B$ associated with $v_{j}, j=1,2$. Then $f(\mathfrak{b}) \cap \triangle f\left(\widehat{v}_{0}\right) f\left(\widehat{v}_{1}\right) f\left(\widehat{v}_{2}\right) \neq \varnothing$, where $\widehat{v}$ denotes the vertex in the domain of $f$ that corresponds to $v \in V(\mathbf{T})$, and $\mathfrak{b}=\left\{\widehat{b}_{1}, \ldots, \widehat{b}_{m}\right\}$.

Proof. Let $\bar{f}: D \rightarrow D$ be an extension of $f$ described earlier. Choose $a \in$ $C\left(v_{1}\right) \cap C\left(v_{2}\right) \cap\left[f\left(\widehat{v}_{1}\right), f\left(\widehat{v}_{2}\right)\right]$. Let $a_{1}$ and $a_{2}$ be the preimages of $a$ under $\bar{f}$ in the star of $\widehat{v}_{1}$ and in the star of $\widehat{v}_{2}$, respectively. Suppose that $\gamma$ is the curve built out of "segments" $\gamma_{1},\left[\widehat{v}_{1}, \widehat{v}_{0}\right],\left[\widehat{v}_{0}, \widehat{v}_{2}\right]$, and $\gamma_{2}$, where $\gamma_{1}$ is the component of $\bar{f}^{-1}\left(\left[a, f\left(\widehat{v}_{1}\right)\right]\right)$ which contains $a_{1}$, and $\gamma_{2}$ is the component of $\bar{f}^{-1}\left(\left[f\left(\widehat{v}_{2}\right), a\right]\right)$ which contains $a_{2}$. Then $\gamma$ is a simple (not closed) curve such that $\bar{f}(\gamma)=\partial\left(\triangle f\left(\widehat{v}_{0}\right) f\left(\widehat{v}_{1}\right) f\left(\widehat{v}_{2}\right)\right)$. By Theorem 3, $\bar{f}: D \backslash \bar{f}^{-1}(f(\mathfrak{b})) \rightarrow D \backslash f(\mathfrak{b})$ is a covering map. In particular, if $\mathscr{D} \subset D \backslash f(\mathfrak{b})$ is a closed topological disk then $\bar{f}^{-1}(\partial \mathscr{D})$ is a disjoint union of simple closed curves $\left\{\beta_{1}, \ldots, \beta_{l}\right\}$ such that $\bar{f} \mid \beta_{j}$ is a homeomorphism onto $\partial \mathscr{D}$ for $j=1, \ldots, l$. Now, if $f(\mathfrak{b}) \cap \triangle f\left(\widehat{v}_{0}\right) f\left(\widehat{v}_{1}\right) f\left(\widehat{v}_{2}\right)=\varnothing$ then $\triangle f\left(\widehat{v}_{0}\right) f\left(\widehat{v}_{1}\right) f\left(\widehat{v}_{2}\right) \subset D \backslash f(\mathfrak{b})$ is a closed topological disk and $\gamma$ is forced to be a simple closed curve, as its image under $\bar{f}$ is $\partial\left(\triangle f\left(\widehat{v}_{0}\right) f\left(\widehat{v}_{1}\right) f\left(\widehat{v}_{2}\right)\right)$, contradiction.

\section{BLASCHKE PRODUCTS}

In this section we will show (Theorem 7.3) that: Any (classical) finite Blaschke product can be approximated uniformly on compacta of $D=\{z:|z|<1\}$ by discrete Blaschke products.

The section is divided into several parts with the last one containing a proof of the above assertion.

1. Classical Blaschke products. We will recall now some facts about finite Blaschke products. Let $\bar{D}$ and $\partial D$ denote the closure and the boundary of the unit disc $D$, respectively. A holomorphic map $F: \bar{D} \rightarrow \bar{D}$ is called a finite Blaschke product if it is of a form $F(z)=c \prod_{i=1}^{N}\left(\frac{z-a_{i}}{1-\bar{a}_{i} z}\right)^{n_{i}}$, where $|c|=1, a_{i} \in$ $D$, and the $n_{i}$ are positive integers. The number $\sum_{i=1}^{N} n_{i}$ is called the degree of $F$. Finite Blascke products have also another characterization: if $F: D \rightarrow D$ is a holomorphic, proper mapping then $F$ is a finite Blaschke product (see $[\mathrm{Ru}])$. Suppose now that $x_{1}, \ldots, x_{m}$ are points in $D$, and that $k_{1}, \ldots, k_{m}$ are positive integers. Then there exists a finite Blaschke product with critical points $x_{1}, \ldots, x_{m}$ of orders $k_{1}, \ldots, k_{m}$, respectively (or equivalently, with the branch set $\left.\left\{\left(x_{1}, k_{1}\right), \ldots,\left(x_{m}, k_{m}\right)\right\}\right)$. Such a Blaschke product is unique up to (postcompositions with) Möbius transformations preserving $D$. 
Let $a, b \in \partial D$ and $a \neq b$. Suppose that $\gamma$ is a simple curve in $D$ with the end points $a$ and $b$. Let $\mathscr{D}$ be a component of $D \backslash \gamma$. Write $F$ for a finite Blaschke product with critical points $x_{1}, \ldots, x_{m}$. Assume that $\left\{x_{1}, \ldots, x_{m}\right\} \cap$ $\mathscr{D}=\varnothing$. Then, since $F$ is a branched covering of $D$ by $D$ (see $[\mathrm{Ru}]$ ), we have the following two facts.

Fact 1.1. If $\left.F\right|_{\mathscr{D}}$ is a homeomorphism onto $F(\mathscr{D})$ then there exists $i, 1 \leq i \leq$ $m$, such that $F\left(x_{i}\right) \notin F(\mathscr{D})$.

Fact 1.2. Let $\gamma^{\prime}=\gamma \backslash\{a, b\}$. If $\left.F\right|_{\mathscr{D}}$ is not a homeomorphism onto $F(\mathscr{D})$ then $F\left(\gamma^{\prime}\right)$ has at least one self-intersection. Moreover, the length of the path $F(\gamma)$ is at least $\inf _{1 \leq i \leq m}\left\{\operatorname{dist}\left(F\left(x_{i}\right), \partial D\right)\right\}$.

2. Subharmonicity of radius functions. Suppose that $\mathbf{T}$ is a triangulation of a disc and $r: V(\mathbf{T}) \rightarrow(0, \infty)$. Then $r(\cdot)$ is said to be subharmonic at $v \in V(\mathbf{T})$ if $r(v)<\frac{1}{n}\left(r\left(v_{1}\right)+\cdots+r\left(v_{n}\right)\right)$, where $v_{1}, \ldots, v_{n}$ are the neighboring vertices of $v . \mathbf{T}$ is called hexagonal if each interior vertex has exactly 6 neighboring vertices and each boundary vertex has at most 6 neighbors. We have the following

Lemma 2.1. Let $r(\cdot)$ be the radius function of a circle packing $P$ of a hexagonal complex $\mathbf{T}$. Then $r(\cdot)$ is subharmonic at interior vertices of $\mathbf{T}$.

Proof. It was shown in [BFP] (see also [R]) that $r(\cdot)$ is subharmonic at any interior vertex with the angle sum $2 \pi$ and with non-overlapping petals of the flower associated with the vertex. However, one can easily verify that the condition that petals are disjoint is irrelevant as long as the angle sum is $2 \pi$. Thus, the radius function $r(\cdot)$ of $P$ is subharmonic at any interior vertex which is not a branch point.

Suppose that $b$ is a branch vertex and $v_{1}, \ldots, v_{6}$ are its neighboring vertices. Recall that the angle sum at $b$ is $4 \pi$. The monotonicity properties of the angle sums imply that if one keeps $r\left(v_{1}\right), \ldots, r\left(v_{6}\right)$ fixed and continuously increases $r(b)$ to $\infty$ then the angle sum at $b$ continuously decreases to 0 . Thus, there is a value $r_{0}(b), r_{0}(b)>r(b)$, such that the angle sum at $b$ given by radii $r_{0}(b), r\left(v_{1}\right), \ldots, r\left(v_{6}\right)$ is equal to $2 \pi$. But then $r(b)<r_{0}(b) \leq \frac{1}{6}\left(r\left(v_{1}\right)+\cdots+r\left(v_{6}\right)\right)$, i.e., the radius function $r(\cdot)$ of $P$ is subharmonic at $b$.

3. Approximation preliminaries. The property that the radius function for a branched circle packing is subharmonic at interior vertices is very special. Since we will need such a property later, from now on we are going to work in this section only with branched circle packings associated with hexagonal complexes, namely, simply connected 2-subcomplexes of the infinite regular hexagonal 2complex. Therefore we need to introduce some notation.

Let $\mathbf{H}$ denote an infinite regular hexagonal 2-complex of mesh 2 with vertices at points $2 k+l(1+\sqrt{3} i), k, l \in \mathbf{Z}$. Write $\mathbf{H}(n)$ for a subcomplex of $\mathbf{H}$ that consists of all faces of $\mathbf{H}$ whose closures are contained in $\{z:|z|<n-1\}$. Let $H(n):=\frac{1}{n} \mathbf{H}(n)$ and let $H^{\prime}(n)$ be a subcomplex of $H(n)$ obtained by removing boundary faces from $H(n)$ (where a face is a boundary face if it has a vertex in bd $H(n))$. We will refer to $\mathbf{H}, \mathbf{H}(n), H(n)$, and $H^{\prime}(n)$ sometimes as simplicial complexes (triangulations) and sometimes as subsets of $\mathbf{C}$, but the 
reader should not have any problems with this duality of notation, as it will be clear from the context to which one we refer.

Let $P_{\mathbf{H}}$ be the regular hexagonal circle packing whose carrier is $\mathbf{H}$. Write $P_{\mathbf{H}(n)}$ for the part of $P_{\mathbf{H}}$ which corresponds to $\mathbf{H}(n) \subset \mathbf{H}$, and $P_{H(n)}$ for $\frac{1}{n} P_{\mathbf{H}(n)}$. We remark that $P_{H(n)}$ is contained in the unit disc.

Fix $n$. Suppose that $B$ is a Bl-type packing for $H(n)$. Let $g$ be the simplicial map from $P_{H(n)}$ to $B$ and $g^{\#}$ be the corresponding ratio map (i.e., $g=\mathscr{S}_{B} \circ \mathscr{S}_{P_{H(n)}^{-1}}^{-1}$ and $\left.g^{\#}=\left(\mathscr{S}_{B} \circ \mathscr{S}_{P_{H(n)}}^{-1}\right)^{\#}\right)$. Using a method analogous to that in Section 3, one constructs an extension of $g$ to a map $\bar{g}: \bar{D} \mapsto \bar{D}$ such that: (1) $\bar{g}=\varphi \circ h$, where $h$ is a self-homeomorphism of $\bar{D}$ and $\varphi$ is a Blaschke product with the branch set $\left\{h\left(b_{1}\right), \ldots, h\left(b_{m}\right)\right\}$, where $\left\{b_{1}, \ldots, b_{m}\right\}$ is the branch set for $B,(2)$ the image under $\bar{g}$ of the "star" (see Section 3 ) of a boundary vertex of $H(n)$ contains the circle of $B$ associated with this vertex. If it is clear from the context which $H(n)$ is the complex for $B$ then we will refer to $g$ and $\bar{g}$ as the simplicial map induced by $B$ and an extended simplicial map associated with $B$, respectively. Finally, we will say that $B$ fixes a point $p \in H(n)$ and maps $q \in H(n)$ to $\mathbf{R}_{+}$if the induced map $g$ fixes $p$ and $g(q) \in \mathbf{R}_{+}$.

Let $A(n)$ be an Andreev circle packing associated with $H(n)$ which fixes the origin and maps $1 / 2$ to $\mathbf{R}_{+}$. Then, by [RS] or [St 1,2], the euclidean radii of boundary circles of $A(n)$ converge uniformly to zero as $n \rightarrow \infty$. Moreover, due to the normalization of $A(n)$, the simplicial maps induced by the $A(n)$ converge uniformly on compacta of $D$ to the identity function. In particular, for fixed $r<1, D(r)=\{z:|z|<r\}$ is mapped under such simplicial functions into $D(\bar{r})$ for sufficiently large $n$, where $\bar{r}=\frac{1+r}{2}$.

Although our goal in this section is to prove the approximation of classical Blaschke products by discrete ones, due to technicalities, most of our work will be in showing that extended simplicial maps approximate classical Blaschke products. Let us be more specific. For a given finite classical Blaschke product $F$ we will construct an appropriate sequence $\left\{B_{F}(n)\right\}$ of Bl-type packings associated with the sequence of complexes $H(n)$. If $F$ is normalized so that $F(0)=0$ and $F(1 / 2) \in \mathbf{R}_{+}$, then the packings $B_{F}(n)$ will fix the origin and map $1 / 2$ to $\mathbf{R}_{+}$. Let $f_{n}$ be an extension of the simplicial map from $P_{H(n)}$ to $B_{F}(n)$ (Figure 4). Let $A(n)$ be as above. Write $\mathbb{F}_{n}$ for the discrete Blaschke product from $A(n)$ to $B_{F}(n)$ and $G_{n}$ for the simplicial map from $P_{H(n)}$ to $A(n)$. We want to show that $\mathbb{F}_{n} \rightarrow F$ uniformly on compacta of $D$. In order to prove this, we will first show that $f_{n} \rightarrow F$ uniformly on compacta of $D$. Then, since $G_{n} \rightarrow \mathrm{id}_{D}$ uniformly on compacta of $D$, we may conclude that $\mathbb{F}_{n}=f_{n} \circ G_{n}^{-1} \rightarrow F$ uniformly on compacta of $D$.

4. Approximating sequences. Let $F$ be a finite Blaschke product with a branch set $\left\{\left(x_{1}, k_{1}\right), \ldots,\left(x_{m}, k_{m}\right)\right\}$. Furthermore, suppose that $F$ is normalized so that $F(0)=0$ and $F(1 / 2) \in \mathbf{R}_{+}$. We remark that such a normalization is merely a convenience, not a restriction for what we are going to do. We need to introduce the following

Definition 4.1. If $\{B(n)\}_{n=N}^{\infty}$ is a sequence of Bl-type packings such that

(1) $B(n)$ is determined by $H(n)$ and a branch set $\left\{v_{i, j}(n)\right\}_{j=1, i=1}^{k_{i}, m}$, fixes the origin, and maps $1 / 2$ to $\mathbf{R}_{+}$, 


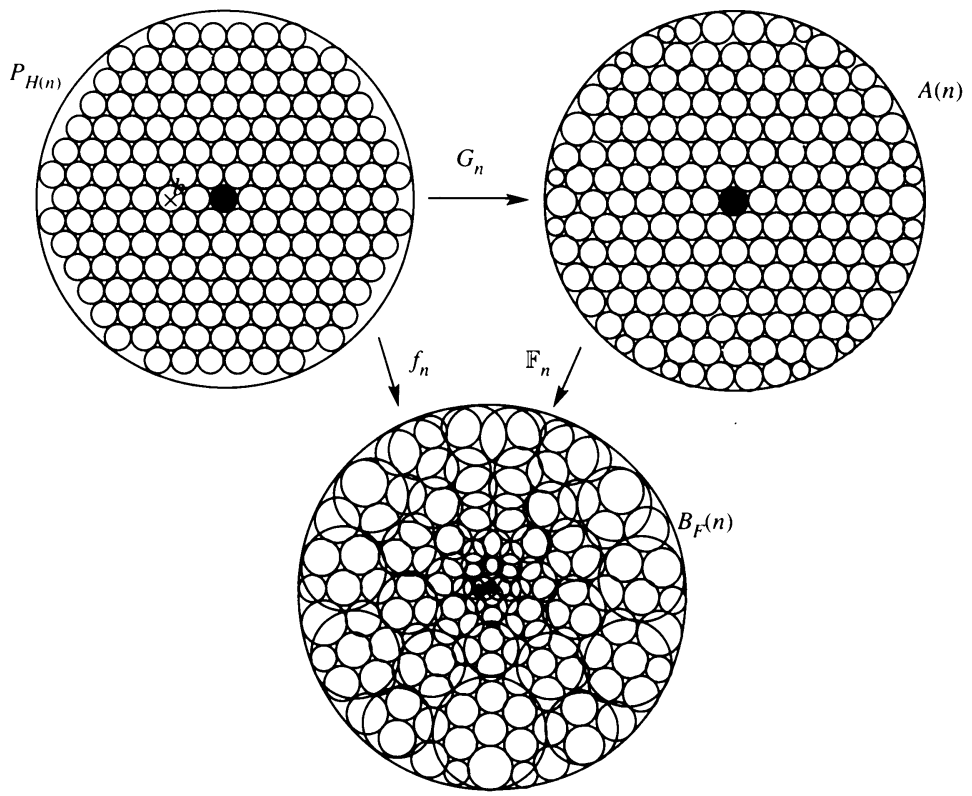

FIGURE 4. Packings and simplicial maps induced by them; $\operatorname{br}\left(B_{F}(n)\right)=\{(b, 1)\}$.

(2) there exists $L \in \mathbf{N}$ such that the combinatorial distance of $v_{i, j}(n)$, for any $n$ and $j=1, \ldots, k_{i}$, to the star in $H(n)$ that contains $x_{i}$ is at most $L$

then $\{B(n)\}$ will be called an approximating sequence of Bl-type packings for $F$.

The reader should be aware of

Remark 4.2. Because of the nature of $\mathbf{H}$ and Theorem 2.5, there always exists an approximating sequence of Bl-type packings for a given $F$. Moreover, a similar definition to Definition 4.1 can be given for $F$ without the normalization by the use of an appropriate Möbius transformation which preserves the unit disc.

Fix an approximating sequence for $F$ and denote it by $\left\{B_{F}(n)\right\}$. Our aim in the reminder of this section is to show that the introduced terminology is justified, namely, that discrete Blaschke products induced by the packings $B_{F}(n)$ converge uniformly to $F$.

5. Packings $B_{F}(n)$. Let $v^{\prime \prime}(n)$ and $\tilde{v}^{\prime \prime}(n)$ denote the centers of the circle associated with vertex $v(n) \in H(n)$ in $A(n)$ in euclidean and hyperbolic geometry, respectively. Write $v^{\prime}(n)$ and $\tilde{v}^{\prime}(n)$ for the centers in $B_{F}(n)$ in euclidean and hyperbolic geometry, respectively. Let $v_{0}(n)$ denote the vertex in $H(n)$ that corresponds to the vertex at the origin in $\mathbf{H}(n)$.

Proposition 5.1. There exists $\bar{r} \in(0,1)$ such that $v_{i, j}^{\prime}(n) \in D(\bar{r})$ for $j=$ $1, \ldots, k_{i}, i=1, \ldots, m$, and any $n$.

Proof. Since $\left\{x_{1}, \ldots x_{m}\right\} \subset D(r)$ for some $r \in(0,1)$, it follows from the choice of the $v_{i, j}(n)$ and Subsection 3 that there is $M \in(0, \infty)$ such that 

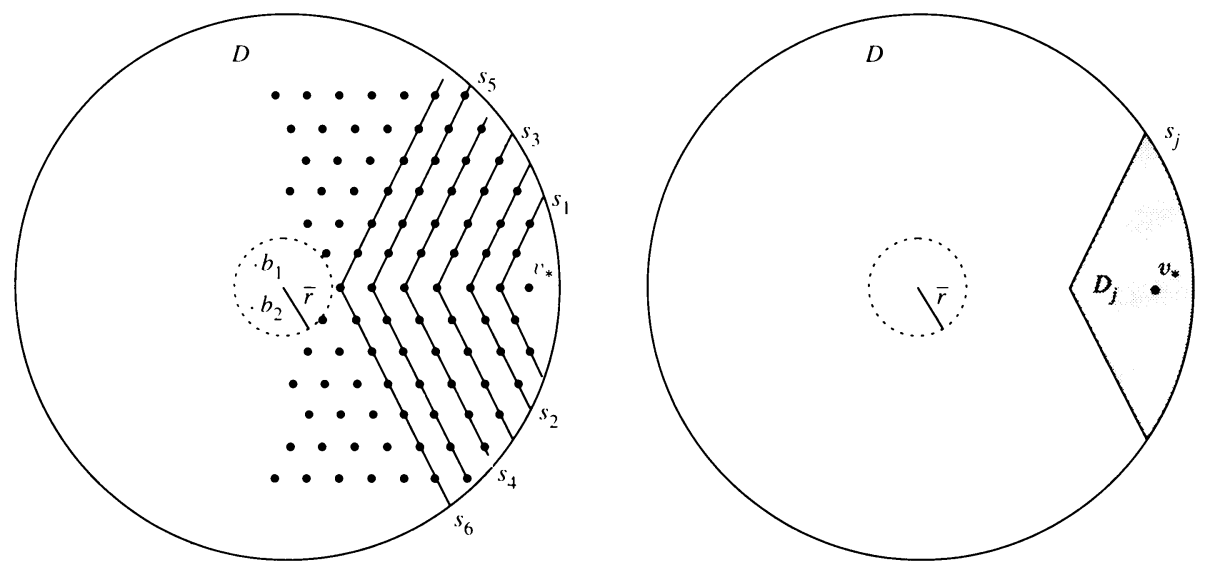

Figure 5. Paths $s_{j}$ and a wedge $D_{j}$.

$\rho\left(0, \tilde{v}_{i, j}^{\prime \prime}(n)\right) \leq M$ for $j=1,2, \ldots, k_{i}, i=1,2, \ldots, m$, and any $n$, where $\rho$ is the hyperbolic metric in $D$. By Theorem 4 of [BSt2], $\rho\left(0, \tilde{v}_{i, j}^{\prime}(n)\right)=$ $\rho\left(\tilde{v}_{0}^{\prime}(n), \tilde{v}_{i, j}^{\prime}(n)\right) \leq \rho\left(\tilde{v}_{0}^{\prime \prime}(n), \tilde{v}_{i, j}^{\prime \prime}(n)\right)=\rho\left(0, \tilde{v}_{i, j}^{\prime \prime}(n)\right)$, and the assertion of our proposition is proved.

We will now prove the main result of this subsection.

Lemma 5.2. The (euclidean) radii of circles in $B_{F}(n)$ go uniformly to zero as $n \rightarrow \infty$.

Proof. The following proof is a generalization of the Rodin-Sullivan length-area argument [RS]. Let $f_{n}$ be an extended simplicial map associated with $B_{F}(n)$

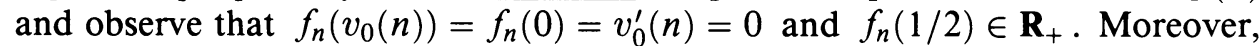
by Theorem 3.4, $f_{n}$ can be written as a composition of a self-homeomorphism of $D$ and a Blaschke product with the values of branch points at $f_{n}\left(v_{i, j}(n)\right)$ 's.

(a) Recall that $f_{n}\left(v_{i, j}(n)\right)=v_{i, j}^{\prime}(n) \in D(\bar{r})$ for any $i, j$, and $n$. Fix $n$. Let $\mathscr{S}_{j}$ be a chain of $n_{j}$ circles in $B_{F}(n)$ of radii $r_{j i}$ that starts and ends at the boundary of $D$. Then, by the Cauchy-Schwarz inequality,

$$
\left(\sum_{i=1}^{n_{j}} r_{j i}\right)^{2} \leq n_{j} \sum_{i=1}^{n_{j}} r_{j i}^{2} \text {. }
$$

Let $l_{j}=2 \sum_{i=1}^{n_{j}} r_{j i}$. Simple computations show

$$
\sum_{j} l_{j}^{2} \frac{1}{n_{j}} \leq 4 \sum_{i, j} r_{j i}^{2} .
$$

Now, consider a boundary vertex $v_{*}$ of $H(n)$ and the collection of edge-paths $s_{j}$ in $H(n)$ that are as in Figure 5 (i.e., $s_{j}$ starts and ends at $\partial D$, it omits the $v_{i, j}(n)$ and it cuts out a "wedge" of $D$, denoted by $D_{j}$, that does not contain the $v_{i, j}(n)$ and has $v_{*}$ in "the middle" of $\left.\partial H(n) \cap D_{j}\right)$.

Let $\mathscr{S}_{j}$ be a chain of circles in $B_{F}(n)$ associated with vertices of $s_{j}$. We will verify the following inequality, 


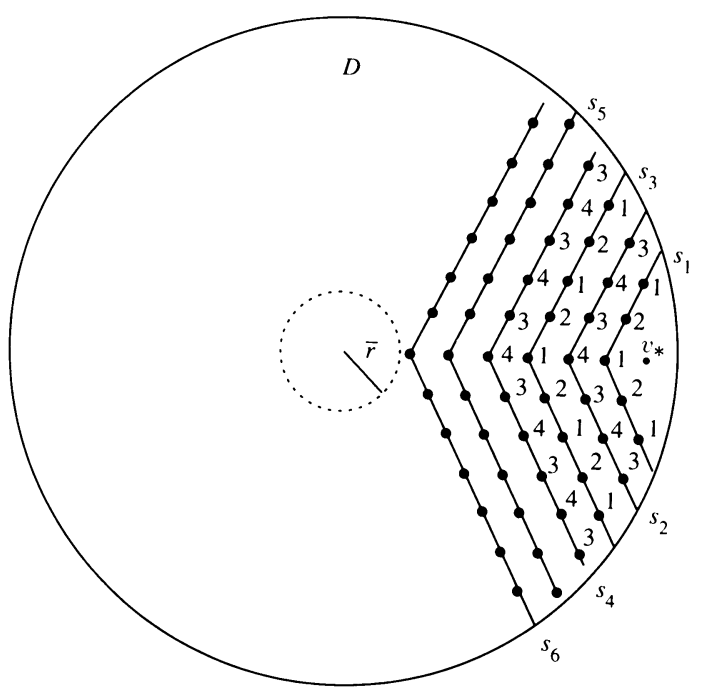

Figure 6. MARKING OF VERTICES

where $r_{n}(\cdot)$ is the radius function of $B_{F}(n)$. By Facts 1.1 and 1.2, we have two possibilities for fixed $j$ :

(1) $\left.f_{n}\right|_{D_{j}}$ is a homeomorphism,

(2) the path $f_{n}\left(s_{j}\right)$ has self-intersection.

In case (1) the circle in $B_{F}(n)$ associated with $v_{*}$ is contained in $f_{n}\left(D_{j}\right)$ and, by Fact $1.1, D \backslash f_{n}\left(D_{j}\right)$ contains at least one value $f_{n}\left(v_{i, j}(n)\right)$. Thus

$$
l\left(f_{n}\left(s_{j}\right)\right) \geq \min \left\{\min _{i, j}\left\{\operatorname{dist}\left(f_{n}\left(v_{i, j}(n)\right), \partial D\right)\right\}, r_{n}\left(v_{*}\right)\right\} \geq \min \left\{1-\bar{r}, r_{n}\left(v_{*}\right)\right\},
$$

where $l\left(f_{n}\left(s_{j}\right)\right)$ denotes the length of the path $f_{n}\left(s_{j}\right)$. Since $l_{j}=l\left(f_{n}\left(s_{j}\right)\right)$, one has $(* *)$ in case (1).

In case (2), by Fact 1.2 we get

$$
l\left(f_{n}\left(s_{j}\right)\right) \geq \min _{i, j}\left\{\operatorname{dist}\left(f_{n}\left(v_{i, j}(n)\right), \partial D\right)\right\} \geq 1-\bar{r} .
$$

Thus $(* *)$ holds in case $(2)$ as well.

Combining inequalities $(*)$ and $(* *)$ one obtains

$$
r_{n}^{2}\left(v_{*}\right) \leq \frac{4}{(1-\bar{r})^{2}}\left(\sum_{i, j} r_{j i}^{2}\right)\left(\sum_{j} \frac{1}{n_{j}}\right)^{-1} .
$$

(b) We will now divide the set of double indices $j i$ into four pairwise disjoint groups $J_{1}, J_{2}, J_{3}, J_{4}$. First let us mark the vertices in the paths $s_{j}$ as in Figure 6, i.e., if $\mathrm{j}$ is odd then every second vertex in $s_{j}$ is marked by 1 and every other vertex in $s_{j}$ is marked by 2 , if $\mathrm{j}$ is even then every second vertex in $s_{j}$ is marked by 3 and every other vertex in $s_{j}$ is marked by 4 .

Then we say that a double index $j i$ is in $J_{k}$ if $r_{j i}$ is associated with the vertex that was marked by $k, k=1,2,3,4$. Notice that the stars in $H(n)$ associated with distinct vertices in $J_{k}$ are disjoint. Moreover, if $C_{n}(v)$ is the circle in $B_{F}(n)$ associated with the vertex $v$ in $H(n)$ then $C_{n}(v)$ is contained 
in $f_{n}(\operatorname{star}(v))$, where $\operatorname{star}(v)$ denotes the star in $H(n)$ associated with the vertex $v$. We also observe that each star in $D$ is mapped homeomorphically by $f_{n}$, except for the stars that correspond to the vertices $v_{i, j}(n)$. Thus, since $f_{n}$ is $M$-to- 1 , we have

$$
\sum_{j i \in J_{k}} \pi r_{j i}^{2} \leq M \pi \text { for } k=1,2,3,4,
$$

where $M=1+\sum_{i=1}^{m} k_{i}$. Hence

$$
\sum_{j, i} \pi r_{j i}^{2} \leq 4 M \pi
$$

The last inequality and $(* * *)$ show that

$$
r_{n}\left(v_{*}\right) \leq \frac{16}{(1-\bar{r})^{2}} M\left(\sum_{j} \frac{1}{n_{j}}\right)^{-1},
$$

which is the extension of Length-Area Lemma of [RS] to the branched case. Now, as $n \rightarrow \infty$ the series $\sum_{j} \frac{1}{n_{j}}$ diverges because $n_{j}=O(j)$, and the last inequality implies that the radii of circles in $B_{F}(n)$ associated with boundary vertices of $H(n)$ go to 0 uniformly in $n$. By Lemma 2.1, the radius function of $B_{F}(n)$ is subharmonic, which gives that the radii of all circles in $B_{F}(n)$ go uniformly to 0 as $n \rightarrow \infty$.

6. Approximation: the special case. In this subsection we will be assuming that all branch points of $F$ are simple. In particular, since $k_{i}=1$ for $i=1, \ldots, m$, we will write $v_{i}(n)$ for $v_{i, 1}(n)$.

Let $f_{n}$ be an extended simplicial map associated with $B_{F}(n)$. We recall that $f_{n}=\varphi_{n} \circ h_{n}$, where $h_{n}: \bar{D} \rightarrow \bar{D}$ is a homeomorphism and $\varphi_{n}$ is a Blaschke product with branch points $h_{n}\left(v_{i}(n)\right)$. Since $f_{n}(0)=0$ and $f_{n}(1 / 2) \in \mathbf{R}_{+}$, we will assume that $h_{n}(0)=0=\varphi_{n}(0)$ and $h_{n}(1 / 2) \in \mathbf{R}_{+}$. (If necessary we use the appropriate Möbius transformation and its inverse composed with $h_{n}$ and $\varphi_{n}$, respectively, to obtain a new factorization of $f_{n}$.) Moreover, we observe that all the Blaschke products $\varphi_{n}$ are of the same degree $m+1$.

In order to keep our notation as clear as possible, we will write $f_{n}\left(v_{i}\right)$ and $h_{n}\left(v_{i}\right)$ for $f_{n}\left(v_{i}(n)\right)$ and $h_{n}\left(v_{i}(n)\right)$, respectively, if there is no reason for confusion.

Let us now recall some facts from the theory of quasiconformal and quasiregular functions that will be stated in two parts $\left(a^{\prime}\right)$ and $\left(a^{\prime \prime}\right)$. All of them can be found in [LV] or [V].

$\left(\mathrm{a}^{\prime}\right)$ If $\mathscr{C} \subset \mathscr{A} \subset \mathbf{R}^{2}$ are such that $\mathscr{A}$ is open and $\mathscr{C}$ is compact then $(\mathscr{A}, \mathscr{C})$ will be called a condenser and $\operatorname{cap}(\mathscr{A}, \mathscr{C})$ will be the conformal capacity of $(\mathscr{A}, \mathscr{C})$. All the $(\mathscr{A}, \mathscr{C})$ condensers we consider will be bounded, which simply means that $\mathscr{A}$ is a bounded set. If $E=(\mathscr{A}, \mathscr{C})$ is a bounded condenser in $\mathbf{R}^{2}$ then

$$
\operatorname{cap} E=M(\Delta(\mathscr{C}, \partial \mathscr{A} ; \mathscr{A})) \text {, }
$$

where $M(\Delta(\mathscr{C}, \partial \mathscr{A} ; \mathscr{A}))$ is the modulus of the curve family $\Delta(\mathscr{C}, \partial \mathscr{A} ; \mathscr{A})$ of paths in $\mathscr{A}$ that connect $\mathscr{C}$ with $\partial \mathscr{A}$. Moreover, if $\mathscr{C}^{\prime} \subset \mathscr{C}, \mathscr{C}^{\prime}$ is compact, then $E^{\prime}:=\left(\mathscr{A}, \mathscr{C}^{\prime}\right)$ is a bounded condenser in $\mathbf{R}^{2}$ and

$$
M(\Gamma) \geq M\left(\Gamma^{\prime}\right),
$$


where $\Gamma=\Delta(\mathscr{C}, \partial \mathscr{A} ; \mathscr{A})$ and $\Gamma^{\prime}=\Delta\left(\mathscr{C}^{\prime}, \partial \mathscr{A} ; \mathscr{A}\right)$. In particular cap $E \geq$ $\operatorname{cap} E^{\prime}$.

If $\mathscr{D}=\left(\mathscr{C}_{0}, \mathscr{C}_{1}\right)$ is a condenser and $\mathscr{C}_{0} \backslash \mathscr{C}_{1}$ is a doubly connected domain then $\mathscr{D}$ is called a ring. The modulus of $\mathscr{D}$ is

$$
\bmod \mathscr{D}=\left(\frac{M\left(\Delta\left(\mathscr{C}_{1}, \partial \mathscr{C}_{0} ; \mathscr{C}_{0} \backslash \mathscr{C}_{1}\right)\right)}{2 \pi}\right)^{-1}
$$

Moreover, $\bmod \mathscr{D}=t$ if and only if $\mathscr{D}$ can be mapped conformally onto the annulus $\left\{z: 1<|z|<e^{t}\right\}$. Also, the capacity $\operatorname{cap} \mathscr{D}\left(\equiv \operatorname{cap}\left(\mathscr{C}_{0}, \mathscr{C}_{1}\right)\right)$ of the ring $\mathscr{D}$ is equal to $M\left(\Delta\left(\mathscr{C}_{1}, \partial \mathscr{C}_{0} ; \mathscr{C}_{0} \backslash \mathscr{C}_{1}\right)\right)$, so

$$
\bmod \mathscr{D}=\frac{2 \pi}{\operatorname{cap} \mathscr{D}} \text {. }
$$

If $\mu(r):=\bmod ((D,[0, r]))$ then $\mu(r)$ is continuous in $(0,1)$, monotonically decreasing, and $\lim _{r \rightarrow 1} \mu(r)=0, \lim _{r \rightarrow 0} \mu(r)=\infty$. In addition one has

Modulus Theorem. If the ring $\mathscr{D}$ separates the points 0 and $r$ from the circle $\{z:|z|=1\}$ then $\bmod \mathscr{D} \leq \mu(r)$.

$\left(\mathbf{a}^{\prime \prime}\right)$ Let $f: G \rightarrow \mathbf{R}^{2}$ be a continuous function. We say that $(\mathscr{A}, \mathscr{C})$ is a normal condenser for $f$ if $(\mathscr{A}, \mathscr{C})$ is a condenser such that $\overline{\mathscr{A}} \subset G$ and $f(\partial \mathscr{A})=\partial f(\mathscr{A})$. If $f$ is non-constant and quasiregular then

$$
\operatorname{cap}(\mathscr{A}, \mathscr{C}) \leq K_{0}(f) N(f, \mathscr{A}) \operatorname{cap}(f(\mathscr{A}, \mathscr{C})),
$$

for all normal condensers $(\mathscr{A}, \mathscr{C})$ for $f$ in $G$, where $K_{0}(f)$ denotes outer dilatation of $f, N(f, \mathscr{A})$ is the maximal number of preimagies in $\mathscr{A}$ of any point from $f(\mathscr{A})$, and $f(\mathscr{A}, \mathscr{C})=(f(\mathscr{A}), f(\mathscr{C}))$. In particular, if $f$ is a Blaschke product of degree $M$ then

$$
\operatorname{cap}(D, \mathscr{C}) \leq M \operatorname{cap}(D, f(\mathscr{C})) \text {, }
$$

for any compact subset $\mathscr{C}$ of $D$. Moreover, if $\mathscr{C}^{\prime}$ is simply connected, $\overline{\mathscr{C}} \overline{\mathscr{C}}^{\prime} \mathrm{C}$ $D$, and $\mathscr{C}$ is a component of $f^{-1}\left(\mathscr{C}^{\prime}\right)$, then

$$
\bmod \mathscr{D}_{2} \leq M \bmod \mathscr{D}_{1}
$$

where $\mathscr{D}_{1}=(D, \mathscr{C})$ and $\mathscr{D}_{2}=\left(D, \mathscr{C}^{\prime}\right)$.

After all these preliminaries, we return now to our functions $h_{n}$ and $\varphi_{n}$.

(a) Recall that, by Proposition 5.1, the images $f_{n}\left(v_{i}\right)$ of branch points $h_{n}\left(v_{i}\right)$ of $\varphi_{n}$ are in $B(0, \bar{r})$, where $B\left(z_{0}, \varrho\right)=\left\{z:\left|z-z_{0}\right|<\varrho\right\}$. We will now use Kquasiregular arguments for $\mathrm{K}=1$ to show that the sets $\varphi_{n}^{-1}\left(f_{n}\left(v_{i}\right)\right)$ are contained in a compact subset of $D$. Since each $\varphi_{n}$ is a Blaschke product of degree $m+1$, by part $\left(\mathbf{a}^{\prime \prime}\right)$ we get

$$
-\ln \bar{r}=\bmod (D, B(0, \bar{r})) \leq(m+1) \bmod \left(D, \varphi_{n}^{-1}(B(0, \bar{r}))\right) .
$$

The Modulus Theorem implies that there exists $\delta>0$ such that $\varphi_{n}^{-1}(B(0, \bar{r})) \subset$ $B(0,1-\delta)$ for any $n$. Thus we have

$$
\exists \delta>0 \ni \varphi_{n}^{-1}\left(f_{n}\left(v_{i}\right)\right) \subset B(0,1-\delta) \text { for any } n \text { and } i=1,2, \ldots, m \text {. }
$$

In particular, all the points $h_{n}\left(v_{i}\right)$ are contained in $B(0,1-\delta)$ for any $n$.

(b) Suppose that $\epsilon>0$. Let $d_{i}^{\epsilon}(n):=\sup \left\{\operatorname{diam} \mathscr{C}_{i}^{\epsilon}(n)\right\}$, where sup is taken over all components $\mathscr{C}_{i}^{\epsilon}(n)$ of $\varphi_{n}^{-1}\left(B\left(f_{n}\left(v_{i}\right), \epsilon\right)\right)$ (Figure 7). Let $d^{\epsilon}(n):=$ 


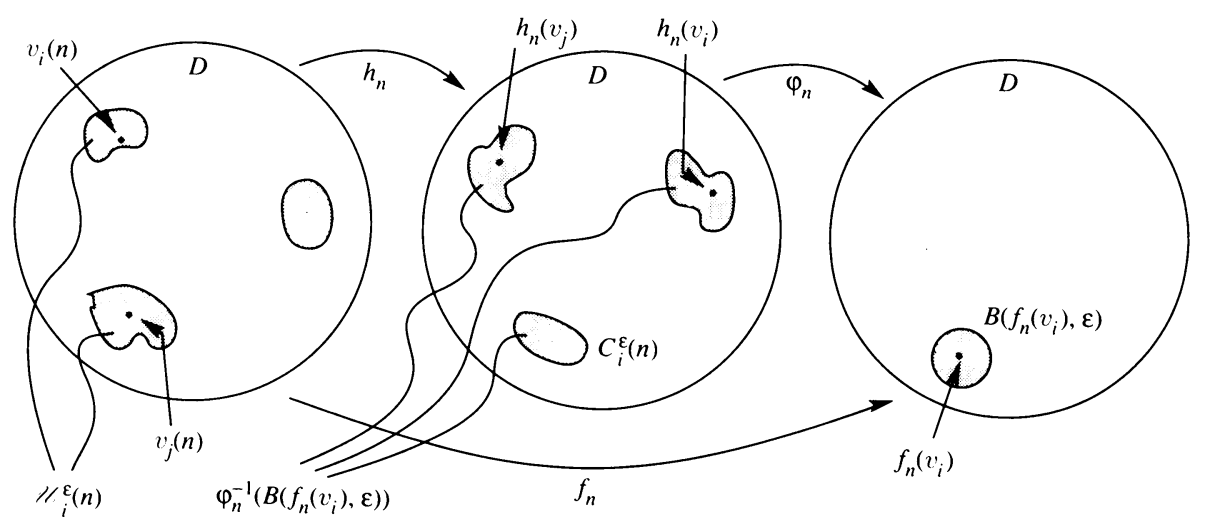

FIGURE 7. Mappings and sets

$\sup _{i}\left\{d_{i}^{\epsilon}(n)\right\}$. Once again, by quasiregular arguments and the fact that images of branch points of functions $\varphi_{n}$ are in $B(0, \bar{r})$ we obtain that $d^{\epsilon}(n) \rightarrow 0$ as $\epsilon \rightarrow 0$ uniformly in $n$ (i.e., for any $\sigma>0$ there exists $\epsilon(\sigma)$ such that $d^{\epsilon}(n)<\sigma$ for any $n$ and any $\left.\epsilon, \epsilon<\epsilon(\sigma)\right)$. $h_{n}$.

(c) We will now prove a lemma about quasiconformality of the functions

Lemma 6.1. Let $\mathscr{U}_{i}^{\epsilon}(n)$ be the union of all components of $f_{n}^{-1}\left(B\left(f_{n}\left(v_{i}\right), \epsilon\right)\right)$ that have non-empty intersection with the set $\left\{v_{j}(n): j=1,2, \ldots, m\right\}$. Let $H_{\epsilon}(n):=H^{\prime}(n) \backslash \bigcup_{i} \overline{\mathscr{U}_{i}^{\epsilon}(n)}$. Then for any $\epsilon>0$ there exists $N_{\epsilon}$ such that $h_{n}$ is $K$-quasiconformal in $H_{\epsilon}(n)$ for any $n>N_{\epsilon}$, where $K$ does not depend on $n$ or $\epsilon$.

Proof. Let $\epsilon>0$. Let $S_{k}(v, n)$ denote the simplicial complex of faces of $k$ generations in $H(n)$ around vertex $v \in H^{\prime}(n)$. By Lemma 5.2, there exists $N_{\epsilon}$ such that for any $n>N_{\epsilon}$

$$
f_{n}\left(S_{3}(v, n)\right) \cap\left\{f_{n}\left(v_{i}\right)\right\}_{i=1}^{m}=\varnothing,
$$

for any vertex $v \in H^{\prime}(n) \backslash \bigcup_{i} \overline{f_{n}^{-1}\left(B\left(f_{n}\left(v_{i}\right), \epsilon\right)\right)}$. Thus, according to Lemma 3.5, for any vertex $v \in H^{\prime}(n) \backslash \bigcup_{i} \overline{f_{n}^{-1}\left(B\left(f_{n}\left(v_{i}\right), \epsilon\right)\right)}$ no flower in $B_{F}(n)$ associated with a vertex of $S_{1}(v, n)$ has overlapping petals. Hence, the Ring Lemma in [RS] (applied to $f_{n} \mid S_{3}(v, n)$ ) implies the existence of a constant $\mathbf{K}$ (associated with the complex $\mathbf{H}$ ) such that $h_{n}$ restricted to $H^{\prime}(n) \backslash \bigcup_{i} \overline{f_{n}^{-1}\left(B\left(f_{n}\left(v_{i}\right), \epsilon\right)\right)}$ is $\mathrm{K}$-quasiconformal for any $n, n>N_{\epsilon}$.

Let $\mathscr{V}_{i}^{\epsilon}(n)$ denote a component of $f_{n}^{-1}\left(B\left(f_{n}\left(v_{i}\right), \epsilon\right)\right) \cap H_{\epsilon}(n)$. To complete the proof of the lemma we only need to show now that there exists $N_{\epsilon}$ such that $\left.f_{n}\right|_{\mathscr{V}_{i}^{\epsilon}(n)}$ is K-quasiconformal for $n>N_{\epsilon}$ and $i=1, \ldots, m$.

A property of Blaschke products tells us that $\left.\varphi_{n}\right|_{h_{n}\left(\mathscr{V}_{i}(n)\right)}$ is a homeomorphism, thus $\left.f_{n}\right|_{\mathscr{V}_{i}(n)}$ is a homeomorphism. Lemma 3.5 and Lemma 5.2 imply that there exists $N_{\epsilon}$ such that the following hold:

(1) If $n>N_{\epsilon}$ and $p \in H^{\prime}(n)$ such that $f_{n}(p) \in D \backslash \bigcup_{i} B\left(f_{n}\left(v_{i}\right), \epsilon / 2\right)$ then no flower in $B_{F}(n)$ associated with any vertex of $S_{10}\left(v_{p}, n\right)$ has overlapping petals, where $v_{p}$ is a vertex in $H^{\prime}(n)$ closest to the point $p$, 
(2) If $n>N_{\epsilon}$ and $p \in H^{\prime}(n)$ such that $f_{n}(p) \in B\left(f_{n}\left(v_{i}\right), \epsilon / 2\right)$ for some $i$ then $f_{n}\left(S_{4}\left(v_{p}, n\right)\right) \subset B\left(f_{n}\left(v_{i}\right), \epsilon\right)$, where $v_{p}$ is defined as in (1).

Let $w$ be a vertex in $\mathscr{V}_{i}^{\epsilon}(n)$, then:

(1) If $n>N_{\epsilon}$ and $w$ is such that $S_{3}(w, n) \subset \mathscr{V}_{i}^{\epsilon}(n)$ then there are no overlapping circles in $B_{F}(n)$ associated with vertices of $S_{2}(w, n)$ because $\left.f_{n}\right|_{S_{3}(w, n)}$ is a homeomorphism,

(2) If $n>N_{\epsilon}$ and $w$ is such that $f_{n}(w) \in D \backslash \bigcup_{i} B\left(f_{n}\left(v_{i}\right), \epsilon / 2\right)$ then, by (1), no flower in $B_{F}(n)$ associated with any vertex of $S_{3}(w, n)$ has overlapping petals,

(3) If $n>N_{\epsilon}$ and there exists $i^{\prime}$ such that $f_{n}(w) \in B\left(f_{n}\left(v_{i^{\prime}}\right), \epsilon / 2\right)$ then $w \in \mathscr{C}$ for some component $\mathscr{C}$ of $f_{n}^{-1}\left(B\left(f_{n}\left(v_{i^{\prime}}\right), \epsilon\right)\right)$. By (2), $S_{3}(w, n) \subset \mathscr{C}$. Moreover, $\mathscr{C}$ does not contain any of $v_{i}(n)$ 's, otherwise, according to the definition of $H_{\epsilon}(n), \mathscr{C} \cap H_{\epsilon}(n)=\varnothing$ and we get a contradiction with the fact that $w \in \mathscr{C} \cap H_{\epsilon}(n)$. In particular, $\left.f_{n}\right|_{\mathscr{C}}$ is a homeomorphism and so is $\left.f_{n}\right|_{S_{3}(w, n)}$. Thus there are no overlapping circles in $B_{F}(n)$ associated with vertices of $S_{2}(w, n)$.

Let $p$ be a point in $\mathscr{V}_{i}^{\epsilon}(n)$ but not a vertex. Let $w_{p}$ be a vertex in $H^{\prime}(n)$ closest to the point $p$, then:

(4) The cases (1)-(3), as above, follow for $w$ replaced by $w_{p}$.

From the cases (1)-(4), the fact that $p \in \operatorname{star}\left(w_{p}\right)$, and the Ring Lemma in [RS], we obtain that $\left.h_{n}\right|_{\mathscr{V}_{i}^{\epsilon}(n)}$ is K-quasiconformal for $n>N_{\epsilon}$.

(d) In this part we will establish properties of the families $\left\{\varphi_{n}\right\}$ and $\left\{h_{n}\right\}$.

We begin with the family $\left\{\varphi_{n}\right\}$. Observe that $\left\{\varphi_{n}\right\}$ is a normal family, and each $\varphi_{n}$ is a Blaschke product of degree $m+1$. Thus, there exists a subsequence $\left\{\varphi_{n_{k}}\right\}$ that converges uniformly on compact subsets of $D$ to a function $\varphi$. From part (a) and the Hurwitz's theorem it follows that $\varphi$ is a Blaschke product of degree $m+1$ with branch points $b_{1}, b_{2}, \ldots, b_{l} \in B(0, \bar{r})$. We will now prove

Lemma 6.2. If $\left\{\varphi_{n_{k}}\right\}$ and $b_{1}, \ldots, b_{l}$ are defined as above then $l=m$.

Proof. Since $\varphi_{n}$ 's have $m$ simple branch points, it follows that $1 \leq l \leq m$. Thus, if $m=1$ there is nothing to prove, so let us assume that $m>1$. From the definition of $\varphi$ we get that $\left\{b_{1}, \ldots, b_{l}\right\}$ is the limit set of the set $\left\{h_{n_{k}}\left(v_{1}\right), \ldots, h_{n_{k}}\left(v_{m}\right)\right\}_{k=0}^{\infty}$ and, without loss of generality, we may assume that there exist $1 \leq t_{1}<\cdots<t_{l} \leq m$ such that $\lim _{k \rightarrow \infty} h_{n_{k}}\left(v_{i}\right)=b_{j}$ for $i=t_{j-1}+$ $1, \ldots, t_{j}$. In order to prove that $l=m$ one has to show that $\lim _{k \rightarrow \infty} h_{n_{k}}\left(v_{i}\right) \neq$ $\lim _{k \rightarrow \infty} h_{n_{k}}\left(v_{i^{\prime}}\right)$ whenever $i \neq i^{\prime}$.

Let $\mathscr{C}_{i}^{\epsilon}(n)$ be as in part (b). Let $\tilde{\mathscr{C}}_{i}^{\epsilon}(n)$ denote $\mathscr{C}_{i}^{\epsilon}(n)$ that has non-empty intersection with the set $\left\{h_{n}\left(v_{j}\right)\right\}_{j=1}^{m}$. Write $\tilde{d}_{i}^{\epsilon}(n):=\sup \left\{\operatorname{diam} h_{n}^{-1}\left(\tilde{\mathscr{C}}_{i}^{\epsilon}(n)\right)\right\}$, where the sup is taken over all $\tilde{\mathscr{C}}_{i}^{\epsilon}(n)$ 's.

Proposition 6.3. If $\tilde{d}_{i}^{\epsilon}(n)$ is defined as above then

$$
\forall \sigma>0 \exists \epsilon_{\sigma} \forall \epsilon<\epsilon_{\sigma} \exists N_{\epsilon} \forall n>N_{\epsilon} \forall i \quad \tilde{d}_{i}^{\epsilon}(n)<\sigma .
$$

Proof. Since, by Lemma 5.2, $f_{n}\left(\partial H^{\prime}(n)\right)$ approaches $\partial D$ uniformly as $n \rightarrow \infty$ (i.e., $f_{n}\left(\partial H^{\prime}(n)\right)$ is in a small neighborhood of $\partial D$ for large $\left.n\right)$, the Schwarz lemma applied to $\varphi_{n}$ shows that $\partial\left(h_{n}\left(H^{\prime}(n)\right)\right)=h_{n}\left(\partial H^{\prime}(n)\right)$ approaches $\partial D$ 
uniformly as $n \rightarrow \infty$. Thus, we may assume without loss of generality that $B(0,1-\delta / 2) \subset h_{n}\left(H^{\prime}(n)\right)$ for any $n$, where $\delta$ is as in part (a) (i.e., $\delta$ is such that $h_{n}\left(v_{i}\right)$ is in $B(0,1-\delta)$ for any $i$ and $\left.n\right)$. The last observation and part (b) show that there exists $\epsilon_{0}$ such that $\tilde{\mathscr{C}}_{i}^{\epsilon}(n) \subset B(0,1-\delta / 2)$ for any $i$, $n$, and $\epsilon<\epsilon_{0}$.

Let $\mathscr{C}^{\epsilon}(n):=\bigcup \tilde{\mathscr{C}}_{i}^{\epsilon}(n)$ and $\widetilde{E}^{\epsilon}(n):=\left(h_{n}\left(H^{\prime}(n)\right), \overline{\mathscr{C}^{\epsilon}(n)}\right)$, where $\overline{\mathscr{C}^{\epsilon}(n)}$ denotes the closure of $\mathscr{C}^{\epsilon}(n)$. Then $\tilde{E}^{\epsilon}(n)$ is a bounded condenser and, by quasiregular arguments, part (b), and above remarks, cap $\widetilde{E}^{\epsilon}(n) \rightarrow 0$ as $\epsilon \rightarrow 0$ uniformly in $n$.

Let $\mathscr{U}^{\epsilon}(n):=\bigcup_{i} \mathscr{U}_{i}^{\epsilon}(n)$. Using the notation of Lemma 6.1 , we observe that $h_{n}\left(\overline{\mathscr{U}^{\epsilon}(n)}\right)=\overline{\mathscr{C}^{\epsilon}(n)}$. Writing $E^{\epsilon}(n)$ for the bounded condenser $\left(H^{\prime}(n), \overline{\mathscr{U}^{\epsilon}(n)}\right)$, we have by part $\left(\mathbf{a}^{\prime}\right)$,

$$
\operatorname{cap} E^{\epsilon}(n) \leq \operatorname{Kcap} \tilde{E}^{\epsilon}(n) \text { for } n>N_{\epsilon},
$$

where $N_{\epsilon}$ is as in Lemma 6.1. Thus

$$
\forall \sigma>0 \exists \epsilon_{\sigma} \forall \epsilon<\epsilon_{\sigma} \exists N_{\epsilon} \forall n>N_{\epsilon} \operatorname{cap} E^{\epsilon}(n)<\sigma .
$$

Since $\widetilde{E}^{\epsilon}(n)$ is a bounded condenser for any $i, n$, and $\epsilon<\epsilon_{0}$, so is the ring $\left(h_{n}\left(H^{\prime}(n)\right), \overline{\tilde{\mathscr{C}}_{i} \epsilon}(n)\right)$ and, by part $\left(\mathrm{a}^{\prime}\right)$,

$(* * *) \quad \operatorname{cap}\left(H^{\prime}(n), h_{n}^{-1}\left(\overline{\tilde{\mathscr{C}}_{i}^{\epsilon}(n)}\right)\right) \leq \operatorname{cap} E^{\epsilon}(n)$ for any $i, n$, and $\epsilon<\epsilon_{0}$.

The Modulus Theorem, $(* *)$, and $(* * *)$ imply the assertion $(\Omega)$.

Proposition 6.4. $\lim _{k \rightarrow \infty} h_{n_{k}}\left(v_{i}\right)=b_{p}$ if and only if $\lim _{k \rightarrow \infty} h_{n_{k}}\left(x_{i}\right)=b_{p}$, where $x_{i}$ is one of the branch points of $F$.

Proof. The definition of $v_{i}(n)$ and the fact that the mesh of $H(n)$ goes to 0 as $n \rightarrow \infty$ imply that $v_{i}(n) \rightarrow x_{i}$ as $n \rightarrow \infty$ for $1 \leq i \leq m$. Now Proposition 6.3 gives

$$
\forall \sigma>0 \exists \epsilon_{\sigma} \exists N_{\sigma} \forall n>N_{\sigma} \quad \bigcup_{i} \mathscr{U}_{i}^{\epsilon_{\sigma}}(n) \subset \bigcup_{i=1}^{m} B\left(x_{i}, \sigma\right),
$$

where $\mathscr{U}_{i}^{\epsilon}(n)$ is defined as in Lemma 6.1. Let

$$
\chi=\min \left\{\min _{i \neq j}\left|x_{i}-x_{j}\right|, \min _{1 \leq i \leq m} \operatorname{dist}\left(x_{i}, \partial D\right)\right\} .
$$

Suppose that $\sigma<\chi / 100$. Let $\epsilon_{\sigma}$ and $N_{\sigma}$ be as in $(*)$. We may again assume without loss of generality that $\bigcup_{i} B\left(x_{i}, \chi / 2\right) \subset H^{\prime}(n)$ for any $n$. Let

$$
\mathscr{D}_{i}(\sigma):=\left(B\left(x_{i}, \chi / 2\right), \overline{B\left(x_{i}, \sigma\right)}\right) \text {. }
$$

Then $\mathscr{D}_{i}(\sigma) \subset H^{\prime}(n) \backslash \bigcup_{i=1}^{m} B\left(x_{i}, \sigma\right)$ for $n>N_{\sigma}$, and by $(*)$ one has

$$
\mathscr{D}_{i}(\sigma) \subset H_{\epsilon_{\sigma}}(n) \text {. }
$$

Lemma 6.1 implies that for every $\sigma, \sigma<\chi / 100$, the homeomorphism $h_{n}$ is Kquasiconformal in $\mathscr{D}_{i}(\sigma)$ for sufficiently large $n$, so we can assume, by making $N_{\sigma}$ larger if necessary, that $h_{n}$ is K-quasiconformal in $\mathscr{D}_{i}(\sigma)$ for $n>N_{\sigma}$. In 
particular, $\bmod \mathscr{D}_{i}(\sigma) \leq \mathrm{K} \bmod h_{n}\left(\mathscr{D}_{i}(\sigma)\right)$ for $n>N_{\sigma}$, and there exists $K_{\sigma}$ such that

$$
\bmod \mathscr{D}_{i}(\sigma) \leq \mathrm{K} \bmod h_{n_{k}}\left(\mathscr{D}_{i}(\sigma)\right) \text { for } k>K_{\sigma} .
$$

Since the rings $\mathscr{D}_{i}(\sigma)$ are such that $\lim _{\sigma \rightarrow 0} \bmod \mathscr{D}_{i}(\sigma)=\infty$, the Modulus Theorem and $(* *)$ give

$$
\forall \tau>0 \exists \sigma_{\tau} \forall \sigma<\sigma_{\tau} \exists K_{\sigma} \quad \operatorname{diam} h_{n_{k}}\left(B\left(x_{i}, \sigma\right)\right)<\tau \text { for } k>K_{\sigma} .
$$

As $\lim _{n \rightarrow \infty} v_{i}(n)=x_{i}$, the last shows that $\lim _{k \rightarrow \infty}\left|h_{n_{k}}\left(v_{i}\right)-h_{n_{k}}\left(x_{i}\right)\right|=0$. This finishes the proof of our proposition.

Proposition 6.5. $\lim _{k \rightarrow \infty} h_{n_{k}}\left(x_{i}\right) \neq \lim _{k \rightarrow \infty} h_{n_{k}}\left(x_{i^{\prime}}\right)$ for $i \neq i^{\prime}$.

Proof. Suppose that the assertion of the proposition is not true. Then, without loss of generality, we can assume that $\lim _{k \rightarrow \infty} h_{n_{k}}\left(x_{1}\right)=\lim _{k \rightarrow \infty} h_{n_{k}}\left(x_{2}\right)$ and, by Propostion $6.4, \lim _{k \rightarrow \infty} h_{n_{k}}\left(x_{1}\right)=b_{1}$. Part (b) and the definitions of $\tilde{\mathscr{C}}_{i}^{\epsilon}(n)$ and $\mathscr{U}_{i}^{\epsilon}(n)$ imply

$$
\forall \sigma>0 \exists \epsilon_{\sigma}, K_{\sigma} \forall k>K_{\sigma} \bigcup_{i} h_{n_{k}}\left(\mathscr{U}_{i}^{\epsilon_{\sigma}}\left(n_{k}\right)\right) \subset \bigcup_{i=1}^{l} B\left(b_{i}, \sigma\right) .
$$

Let

$$
\tilde{\chi}=\min \left\{\min _{i \neq j}\left|b_{i}-b_{j}\right|, \min _{1 \leq i \leq l} \operatorname{dist}\left(b_{i}, \partial D\right)\right\} .
$$

Since $\varphi_{n_{k}}$ fixes 0 and $\varphi_{n_{k}}\left(h_{n_{k}}\left(\partial H^{\prime}\left(n_{k}\right)\right)\right)=f_{n_{k}}\left(\partial H^{\prime}\left(n_{k}\right)\right)$ approaches $\partial D$ as $k \rightarrow \infty$, the Schwarz lemma applied to $\varphi_{n_{k}}$ shows that $\partial\left(h_{n_{k}}\left(H^{\prime}\left(n_{k}\right)\right)\right)$ approaches $\partial D$ as $k \rightarrow \infty$. Therefore, we can assume without loss of generality that $B\left(b_{1}, \tilde{\chi} / 2\right) \subset h_{n_{k}}\left(H^{\prime}\left(n_{k}\right)\right)$ for any $k>K_{\sigma}$ and $\sigma<\tilde{\chi} / 100$, where $K_{\sigma}$ is defined as in $(*)$. Moreover, for any $\sigma<\tilde{\chi} / 100$ and $k>K_{\sigma}$ one has

$$
\widetilde{\mathscr{D}}_{1}(\sigma):=\left(B\left(b_{1}, \tilde{\chi} / 2\right), \overline{B\left(b_{1}, \sigma\right)}\right) \subset h_{n_{k}}\left(H^{\prime}\left(n_{k}\right)\right) \backslash \bigcup_{i=1}^{l} B\left(b_{i}, \sigma\right) \subset h_{n_{k}}\left(H_{\epsilon_{\sigma}}\left(n_{k}\right)\right) \text {. }
$$

Now Lemma 6.1 implies that for any $\sigma, \sigma<\tilde{\chi} / 100$, the homeomorphism $h_{n_{k}}^{-1}$ is K-quasiconformal in $H_{\epsilon_{\sigma}}\left(n_{k}\right) \supset h_{n_{k}}^{-1}\left(\widetilde{\mathscr{D}}_{1}(\sigma)\right)$ for sufficiently large $k$. Therefore, by making $K_{\sigma}$ large enough if necessary, we can assume that $h_{n_{k}}^{-1}$ is $\mathrm{K}$-quasiconformal in $h_{n_{k}}^{-1}\left(\widetilde{\mathscr{D}}_{1}(\sigma)\right)$ for $k>K_{\sigma}$. Hence

$(* *) \quad \bmod \widetilde{\mathscr{D}}_{1}(\sigma) \leq \operatorname{Kmod} h_{n_{k}}^{-1}\left(\widetilde{\mathscr{D}}_{1}(\sigma)\right)$ for $k>K_{\sigma}$ and $\sigma<\tilde{\chi} / 100$.

Since $\lim _{k \rightarrow \infty} h_{n_{k}}\left(x_{1}\right)=b_{1}=\lim _{k \rightarrow \infty} h_{n_{k}}\left(x_{2}\right)$, for any $\sigma$ there exists $k_{\sigma}$ such that $h_{n_{k}}\left(v_{1}\right), h_{n_{k}}\left(v_{2}\right) \in B\left(b_{1}, \sigma\right)$ for $k>k_{\sigma}$, and without loss of generality we may assume that $K_{\sigma} \geq k_{\sigma}$. In particular, $v_{1}\left(n_{k}\right), v_{2}\left(n_{k}\right) \in h_{n_{k}}^{-1}\left(B\left(b_{1}, \sigma\right)\right)$ for $k>K_{\sigma}$. Now recall that $\lim _{k \rightarrow \infty} v_{1}\left(n_{k}\right)=x_{1}$ and $\lim _{k \rightarrow \infty} v_{2}\left(n_{k}\right)=x_{2}$, hence $\operatorname{diam} h_{n_{k}}^{-1}\left(B\left(b_{1}, \sigma\right)\right)>\chi / 2$ for sufficiently large $k$, say $k>K_{\sigma}$, where $\chi$ is defined as in the proof of Proposition 6.4. The Modulus Theorem shows that for any $\sigma<\tilde{\chi} / 100$

$(* * *) \quad \bmod h_{n_{k}}^{-1}\left(\widetilde{\mathscr{D}}_{1}(\sigma)\right) \leq \operatorname{const}(\tilde{\chi})$ for $k>K_{\sigma}$, 
where const $(\tilde{\chi})$ depends only on $\tilde{\chi}$. Combining $(* *)$ and $(* * *)$, and letting $\sigma \rightarrow 0$, we obtain a contradiction. This finishes the proof of the proposition.

The above proposition completes the proof of Lemma 6.2.

We can summarize the results about subsequences $\left\{\varphi_{n_{k}}\right\}$ and $\left\{h_{n_{k}}\right\}$ which we have obtained so far in the following

Lemma 6.6. The limit function $\varphi$ of the sequence $\left\{\varphi_{n_{k}}\right\}$ is a Blaschke product with simple branch points at $b_{1}, \ldots, b_{m}$, where $b_{i}=\lim _{k \rightarrow \infty} h_{n_{k}}\left(x_{i}\right)$ for $i=$ $1,2, \ldots, m$.

We now turn our attention to quasiconformal properties of the family $\left\{h_{n_{k}}\right\}$.

Let $D_{n}:=\{z:|z|<1-1 / n\} \backslash \bigcup_{i=1}^{m} B\left(x_{i}, 1 / n\right)$ and $\widetilde{D}_{n}:=\{z:|z|<$ $1-1 / n\} \backslash \bigcup_{i=1}^{m} B\left(b_{i}, 1 / n\right)$. Since $\partial H^{\prime}(n)$ tends to $\partial D,(*)$ in the proof of Proposition 6.4 shows that for any $l \in \mathbf{N}$ there exist $\epsilon_{l}$ and $N_{l}$ such that $D_{l} \subset H_{\epsilon_{l}}(n)$ for $n>N_{l}$. In particular, from Lemma 6.1 it follows

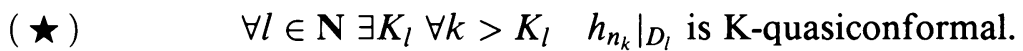

Similarly, $(*)$ in the proof of Proposition 6.5 and the fact that $\partial\left(h_{n_{k}}\left(H^{\prime}\left(n_{k}\right)\right)\right)$ tends to $\partial D$ show that for any $l \in \mathbf{N}$ there exist $\epsilon_{l}$ and $\widetilde{K}_{l}^{\prime}$ such that $\widetilde{D}_{l} \subset$ $h_{n_{k}}\left(H_{\epsilon_{l}}\left(n_{k}\right)\right)$ for $k>\widetilde{K}_{l}^{\prime}$. Thus

$$
\forall l \in \mathbf{N} \exists \widetilde{K}_{l} \forall k>\left.\widetilde{K}_{l} \quad h_{n_{k}}^{-1}\right|_{\widetilde{D}_{l}} \text { is K-quasiconformal. }
$$

The way the functions $h_{n_{k}}$ are defined, $(\star), \widetilde{(\star)}$, and standard quasiconformal arguments show

Lemma 6.7. The family $\left\{h_{n_{k}}\right\}$ of uniformly bounded functions has the following properties:

(1) $\left\{h_{n_{k}}\right\}$ is a normal family in $D \backslash\left\{x_{1}, x_{2}, \ldots, x_{m}\right\}$,

(2) any convergent subsequence of $\left\{h_{n_{k}}\right\}$ tends uniformly on compacta of $D \backslash\left\{x_{1}, x_{2}, \ldots, x_{m}\right\}$ to some $K$-quasiconformal function $h$,

(3) the limit function $h$ is the restriction of a K-quasiconformal homeomorphism $\tilde{h}, \tilde{h}: D \rightarrow D$, with $\tilde{h}\left(x_{i}\right)=b_{i}$ for $i=1,2, \ldots, m$, and

(4) due to the normalization condition on the functions $h_{n_{k}}$, the function $\tilde{h}$ fixes 0 and $\tilde{h}(1 / 2) \in \mathbf{R}_{+}$.

We are now going to prove

Lemma 6.8. Let $\left\{h_{n_{k}^{\prime}}\right\}$ be a convergent subsequence of $\left\{h_{n_{k}}\right\}$. Let $h$ be the limit function of $\left\{h_{n_{k}^{\prime}}\right\}$ and $\tilde{h}$ be the function described as in Lemma 6.7(3). Then $\tilde{h}=\mathrm{id}_{D}$.

Proof. In order to prove our statement we will show that $h$ is 1-quasiconformal, i.e., conformal. Then also $\tilde{h}$ is 1-quasiconformal, and the Riemann mapping theorem implies that $\tilde{h}=\mathrm{id}_{D}$.

Let $z_{0} \in D \backslash\left\{x_{1}, \ldots, x_{m}\right\}$. Choose $\delta>0$ so that $\overline{B\left(z_{0}, \delta\right)} \subset D \backslash\left\{x_{1}, \ldots\right.$, $\left.x_{m}\right\}$. Then $\left.h_{n_{k}^{\prime}}\right|_{\overline{B\left(z_{0}, \delta\right)}}$ converges uniformly to $\left.h\right|_{\overline{B\left(z_{0}, \delta\right)}}$. Since $h\left(z_{0}\right) \in D \backslash$ $\left\{b_{1}, \ldots, b_{m}\right\}, h\left(z_{0}\right)$ is not a branch point of $\varphi$, and there are $\epsilon$ and $k_{0}$ such 
that $\left.\varphi_{n_{k}}\right|_{B\left(h\left(z_{0}\right), \epsilon\right)}$ are 1-to-1 for $k>k_{0}$. The equicontinuity of the normal family $\left\{h_{n_{k}^{\prime}}\right\}$ implies the existence of $\sigma, \sigma \leq \delta$, and $k_{1}, k_{1} \geq k_{0}$, such that $h_{n_{k}^{\prime}}\left(B\left(z_{0}, \sigma\right)\right) \subset B\left(h\left(z_{0}\right), \epsilon\right)$ for $k>k_{1}$. Thus $\left.f_{n_{k}}\right|_{B\left(z_{0}, \sigma\right)}$ is 1-to-1 for $k>k_{1}$.

Now we are ready to make a crucial step in showing that $h$ is 1-quasiconformal. As $k \rightarrow \infty$, we have an ever-increasing number of generations of $H\left(n_{k}^{\prime}\right)$ 's around $z_{0}$ in $B\left(z_{0}, \sigma\right)$ and associated with them univalent (i.e., non-overlapping) hexagonal circle packings that are parts of the corresponding packings $B_{F}\left(n_{k}^{\prime}\right)$. From the Hexagonal Packing Lemma in [RS] we conclude that the quasiconformal distortion of the $f_{n_{k}^{\prime}}$ at $z_{0}$ goes to 0 as $k \rightarrow \infty$. (We remark here that in [RS] circle packings are, by definition, univalent packings.) Hence $f:=\varphi \circ h=\lim _{k \rightarrow \infty}\left(\varphi_{n_{k}^{\prime}} \circ h_{n_{k}^{\prime}}\right)$ has the quasiconformal distortion at $z_{0}$ equal to 0 . As $z_{0}$ was arbitrary, this shows that $f$ is 1-quasiregular and $h$ is 1quasiconformal.

As an immediate consequence of the above lemma we have

Corollary 6.9. The sequence $\left\{h_{n_{k}}\right\}$ converges uniformly on compacta of $D$ to $\operatorname{id}_{D}$.

From Corollary 6.9 and Lemma 6.6 it follows that $x_{i}=b_{i}$. Since $\varphi$ and $F$ are finite Blaschke products with identical branch sets and satisfy $\varphi(0)=F(0)$ and $\varphi(1 / 2), F(1 / 2)>0$, we conclude that $\varphi \equiv F$. Hence $\left\{f_{n_{k}}\right\}$ converges uniformly on compacta of $D$ to $F$. Thus, by standard arguments, the full sequence $\left\{f_{n}\right\}$ converges uniformly on compacta of $D$ to $F$.

Recall that $f_{n}$ is an extension $\bar{F}_{n}$ of the simplicial map $F_{n}$ from $P_{H(n)}$ to $B_{F}(n)$. Therefore, we have shown that the maps $F_{n}$ converge uniformly on compacta of $D$ to $F$.

7. Approximation: the general case. We must discuss how the restriction in Subsection 6 that $F$ has only simple branch points can be removed. The only reason why we did not prove all the results in Subsection 6 in the general case was that we would be overwhelmed by notation, due to double indices for example, that would shadow the essence of the arguments.

In general, suppose $F$ is a finite Blaschke product with a branch set $\left\{\left(x_{1}, k_{1}\right)\right.$, $\left.\ldots,\left(x_{m}, k_{m}\right)\right\}$ (not necessarily simple branch points) such that $F(0)=0$ and $F(1 / 2) \in \mathbf{R}_{+}$. Let $\left\{B_{F}(n)\right\}$ be an approximating sequence of Bl-type packings for $F$ with branch sets $\left\{v_{i, j}(n)\right\}_{j=1, i=1}^{k_{i}, m}$ (see Subsection 4). Let $f_{n}$ be an extended simplicial map associated with $B_{F}(n)$. Then $f_{n}=\varphi_{n} \circ h_{n}$, where $h_{n}: \bar{D} \mapsto \bar{D}$ is a homeomorphism and $\varphi_{n}$ is a Blaschke product with branch points $\left\{h_{n}\left(v_{i, j}(n)\right)\right\}_{j=1, i=1}^{k_{i}, m}$. Moreover, Proposition 5.1 and Lemma 5.2 imply that the results in Subsection $6(\mathrm{a}),(\mathrm{b})$, and (c) hold with the various quantities $v_{i}(n), \mathscr{C}_{i}^{\epsilon}(n), d_{i}^{\epsilon}(n)$, etc., of that subsection, replaced by their doubly-indexed counterparts. As in Subsection $6(\mathrm{~d}),\left\{\varphi_{n}\right\}$ has a convergent subsequence $\left\{\varphi_{n_{k}}\right\}$ with a limit function $\varphi$, and, since all the $v_{i, j}(n)$ are in $B(0, \bar{r}), \varphi$ is a Blaschke product with branch points $b_{1}, \ldots, b_{l} \in B(0, \bar{r})$. We also remark that the degree of $\varphi$ is the same as the degree of each $\varphi_{n_{k}}$. Using the same quasiregular techniques as in the proofs of Propositions 6.3-6.5 one shows

Lemma 7.1. The limit function $\varphi$ of the subsequence $\left\{\varphi_{n_{k}}\right\}$ is a Blaschke product with the branch set $\left\{\left(b_{1}, k_{1}\right), \ldots,\left(b_{m}, k_{m}\right)\right\}$, where $b_{i}=\lim _{k \rightarrow \infty} h_{n_{k}}\left(x_{i}\right)$. 
Finally, since all quasiconformal properties of the family $\left\{h_{n_{k}}\right\}$ discussed in Subsection 6 carry over to our current setting, $\left\{f_{n_{k}}\right\}$ converges uniformly on compacta of $D$ to $F$. Moreover, since $f_{n}$ is an extension $\bar{F}_{n}$ of the simplicial map $F_{n}$ from $P_{H(n)}$ to $B_{F}(n)$, Theorem 1 of [DSt] (see Comments after the proof of the theorem) implies that the functions $F_{n_{k}}^{\#}$ converge uniformly on compacta of $D$ to $\left|F^{\prime}\right|$. Thus we have

Theorem 7.2. Given a finite Blaschke product $F$ normalized by $F(0)=0$ and $F(1 / 2) \in \mathbf{R}_{+}$, let $\left\{B_{F}(n)\right\}$ be an approximating sequence of Bl-type packings for $F$, and write $F_{n}$ for the simplicial map from $P_{H(n)}$ to $B_{F}(n)$. Then functions $F_{n}$ and $F_{n}^{\#}$ converge uniformly on compacta of $D$ to $F$ and $\left|F^{\prime}\right|$, respectively.

An almost immediate consequence of the above result is

Theorem 7.3. Let $F$ be a finite classical Blaschke product. Then there exists a sequence $\left\{\mathbb{F}_{n}\right\}$ of discrete Blaschke products such that functions $\mathbb{F}_{n}$ and $\mathbb{F}_{n}^{\#}$ converge uniformly on compacta of $D$ to $F$ and $\left|F^{\prime}\right|$, respectively.

Proof. Since a discrete (classical) Blaschke product followed by a map $\mathscr{M}(z)=$ $c \frac{z-a}{1-z \bar{a}},|a|<1,|c|=1$, is also a discrete (classical) Blaschke product, we may assume without loss of generality that $F(0)=0$ and $F(1 / 2) \in \mathbf{R}_{+}$. Suppose that $A(n)$ is an Andreev circle packing for $H(n)$ that fixes the origin and maps $1 / 2$ to $\mathbf{R}_{+}$. Let $B_{F}(n)$ be an approximating sequence of Bl-type packings for $F$. Write $\mathbb{F}_{n}$ for the discrete Blaschke product from $A(n)$ to $B_{F}(n)$. Let $F_{n}$ and $G_{n}$ be the simplicial maps from $P_{H(n)}$ to $B_{F}(n)$ and from $P_{H(n)}$ to $A(n)$, respectively. We observe that $\mathbb{F}_{n}=F_{n} \circ G_{n}^{-1}$. By [RS], functions $G_{n}$ converge uniformly on compacta of $D$ to the identity map of $D$ while, by Theorem 7.2, maps $F_{n}$ converge uniformly on compacta of $D$ to $F$. Hence the functions $\mathbb{F}_{n}$ converge uniformly on compacta of $D$ to $F$.

The convergence of maps $\mathbb{F}_{n}^{\#}$ to $\left|F^{\prime}\right|$ is a consequence of Theorem 1 of [DSt].

Before we finish this section let us make four remarks.

Remark 7.4. Theorem 7.3 has already found its application in [DSt] where it was used to prove the branched Schwarz lemma (a classical result due to Z. Nehari) via circle packing.

Remark 7.5. Let $\alpha_{n}: \mathbf{H}(n) \rightarrow \mathbf{H}$ be the natural inclusion and let $\beta_{n}: H(n) \rightarrow \mathbf{H}$ be the map $\alpha_{n} \circ t_{n}$, where $t_{n}: H(n) \rightarrow \mathbf{H}(n)$ is a scaling by multiplication by $n$. Recall that $v_{0}(n)=0$ for every $n$, so $\beta_{n}\left(v_{0}(n)\right)=0$ for every $n$. Suppose that vertices $v_{i}(n), i=1, \ldots, m$, have the same combinatorial location in $H(n)$ with respect to $v_{0}(n)$ for any $n$, i.e., $\beta_{n}\left(v_{i}(n)\right)=\beta_{n^{\prime}}\left(v_{i}\left(n^{\prime}\right)\right)$ for any $n, n^{\prime} \in \mathbf{N}$ and any $i=1,2, \ldots, m$ (in other words, for a fixed $i$, the vertices $\beta_{n}\left(v_{i}(n)\right)$ have fixed location in $\mathbf{H}$ as $n$ changes).

Let $B(n)$ be the Bl-type packing for $H(n)$ with $\operatorname{br}(B(n))=\left\{v_{1}(n), \ldots\right.$, $\left.v_{m}(n)\right\}$ fixing 0 and mapping $1 / 2$ to $\mathbf{R}_{+}$. Then $B(n)$ is an approximating sequence of Bl-type packings for $F(z)=z^{m+1}$. In particular, if $F_{n}$ is the simplicial map from $P_{H(n)}$ to $B(n)$ then the functions $F_{n}$ converge uniformly on compacta of $D$ to the function $z^{m+1}$.

Remark 7.6. Let $F(z)=z^{m}$ and let $\left\{B_{F}(n)\right\}$ be an approximating sequence of Bl-type packings for $F$. Suppose that $0<t_{0}<t_{1}<1$. Then for any $\delta>0$ 
there exists $N_{\delta}$ such that

$$
\frac{m t_{0}^{m-1}-\delta}{m t_{1}^{m-1}+\delta} \leq \frac{r_{n}(w)}{r_{n}(v)} \leq \frac{m t_{1}^{m-1}+\delta}{m t_{0}^{m-1}-\delta} \text { for } n>N_{\delta},
$$

where $v, w$ are vertices of $H(n)$ that are in $\left\{z: t_{0} \leq|z| \leq t_{1}\right\}$, and $r_{n}(\cdot)$ is the radius function of $B_{F}(n)$.

Proof. Since $F_{n}^{\#}(v)=r_{n}(v) /(1 / n)$ for any vertex $v$ in $H(n)$ and $\left|F^{\prime}(z)\right|=$ $m t^{m-1}$ for $|z|=t$, the assertion of our remark follows from Theorem 7.2.

Remark 7.7. Suppose that $\Omega$ is a bounded simply connected domain in $\mathbf{C}$. Let $F: \Omega \rightarrow D$ be a proper analytic map with a branch set $\left\{\left(x_{1}, k_{1}\right), \ldots,\left(x_{m}, k_{m}\right)\right\}$ (i.e., $F=G \circ \omega$, where $\omega: \Omega \rightarrow D$ is a Riemann mapping and $G: D \rightarrow D$ is a finite Blaschke product with the branch set $\left.\left\{\left(\omega\left(x_{1}\right), k_{1}\right), \ldots,\left(\omega\left(x_{m}\right), k_{m}\right)\right\}\right)$. Write $H_{\Omega}(n)$ for a subcomplex of $\frac{1}{n} \mathbf{H}$ which is the union of all closed faces of $\frac{1}{n} \mathbf{H}$ contained in $\Omega$. Let $P_{H(n)}(\Omega)$ be the part of the circle packing $\frac{1}{n} P_{\mathrm{H}}$ which corresponds to the subcomplex $H_{\Omega}(n)$ of $\frac{1}{n} \mathbf{H}$. Define points $v_{i, j}(n)$ and Bl-type packings $B_{F}(n)$ as in Subsection 4 but this time for the complexes $H_{\Omega}(n)$ instead of the $H(n)$. Denote $F_{n}$ the simplicial map from $H_{\Omega}(n)$ to $B_{F}(n)$. Then functions $F_{n}$ and $F_{n}^{\#}$ converge uniformly on compacta of $\Omega$ to $F$ and $\left|F^{\prime}\right|$, respectively.

\section{REFERENCES}

[BFP] I. Bárány, Z. Füredi, and J. Pach, Discrete convex functions and proof of the six circle conjecture of Fejes Tóth, Canad. J. Math 36 (1984), 569-576.

[BSt1] Alan F. Beardon and Kenneth Stephenson, The uniformization theorem for circle packings, Indiana Univ. Math. J. 39 (1990), 1383-1425.

[BSt2] _ The Schwarz-Pick lemma for circle packings, Illinois. J. Math. 141 (1991), 577-606.

[B] Philip L. Bowers, The upper Perron method for labeled complexes, Proc. Cambridge Philos. Soc. 114 (1992), 321-345.

[CR] I. Carter and Burt Rodin, An inverse problem for circle packing and conformal mapping, Trans. Amer. Math. Soc. 334 (1992), 861-875.

[D] Tomasz Dubejko, Infinite branched circle packings and discrete complex polynomials, preprint.

[DSt] Tomasz Dubejko and Kenneth Stephenson, The branched Schwarz lemma: a classical result via circle packing, Michigan Math. J. (to appear).

[G] B. T. Garrett, Circle packings and polyhedral surfaces, Discrete and Comput. Geom. 8 (1992), 429-440.

[LV] Olli Lehto and K.I. Virtanen, Quasiconformal mapping in the plane, 2nd ed., SpringerVerlag, New York, 1973.

[R] Burt Rodin, Schwarz's lemma for circle packings, Invent. Math. 89 (1987), 271-289.

[RS] Burt Rodin and Dennis Sullivan, The convergence of circle packings to the Riemann mapping, J. Differential Geom. 26 (1987), 349-360.

[Ru] Walter Rudin, Function theory in the unit ball of $\mathbf{C}^{n}$, Springer-Verlag, New York, 1980.

[St1] Kenneth Stephenson, Circle packings in the approximation of conformal mappings, Bull. Amer. Math. Soc. 23 (1990), 407-415.

[St2] - A probabilistic proof of Thurston's conjecture on circle packings, preprint.

[T1] William Thurston, The finite Riemann mapping theorem, Invited talk, An International Symposium at Purdue University on the occasion of the proof of the Bieberbach conjecture, March 1985. 
[T2] - The geometry and topology of 3-manifolds, Princeton University Notes, 1980.

[V] Matti Vuorinen, Conformal geometry and quasiregular mappings, Lecture Notes in Math., vol. 1319, Springer-Verlag, 1988.

Department of Mathematics, University of Tennessee, Knoxville, Tennessee 37996 1300

Current address: Mathematical Sciences Research Institute, Berkeley, California 94720

E-mail address: tdubejko@msri.org 